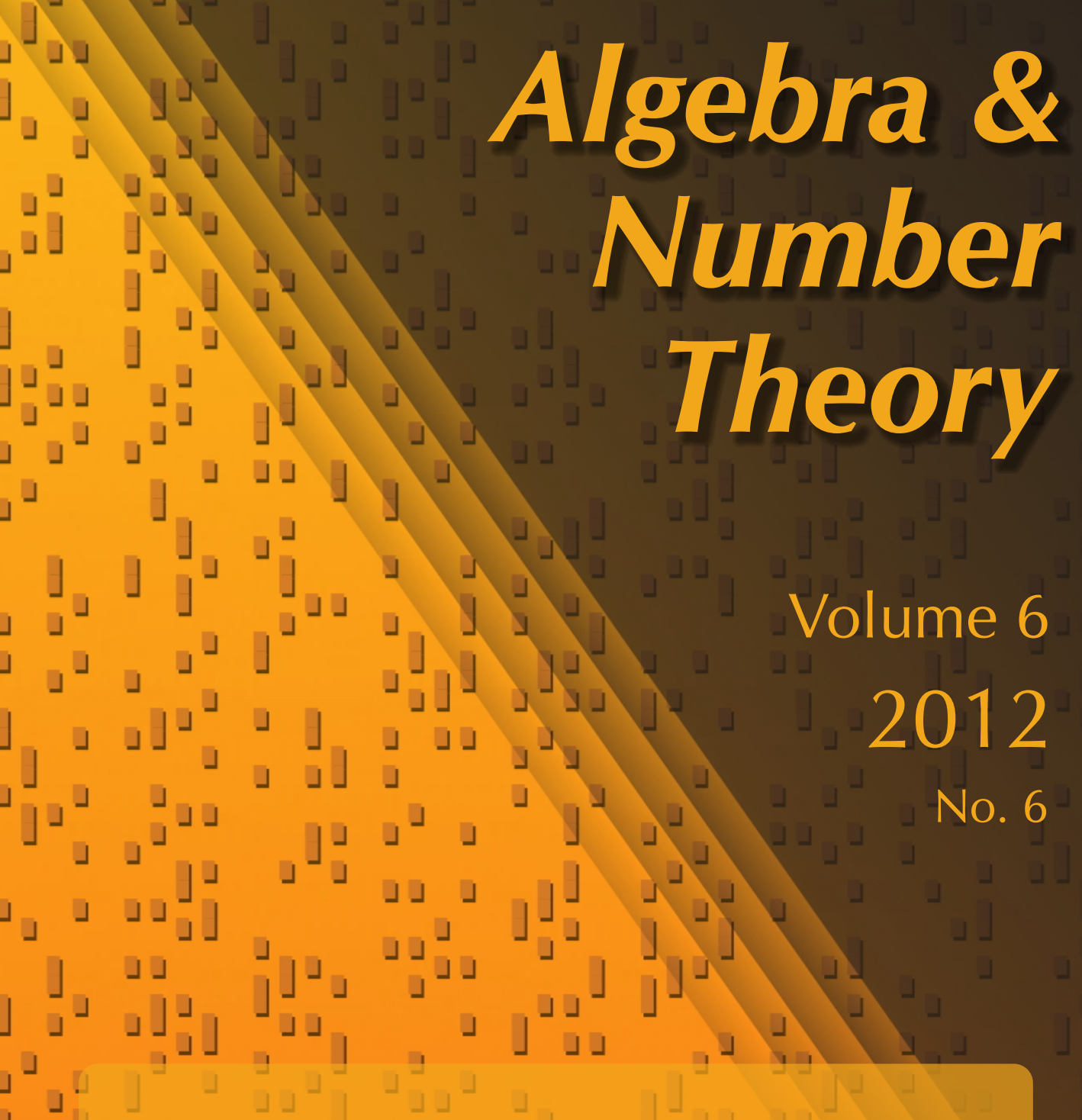

On fusion categories with few irreducible degrees

$\downarrow$ Sonia Natale and Julia Yael Plavnik

\lrcorner

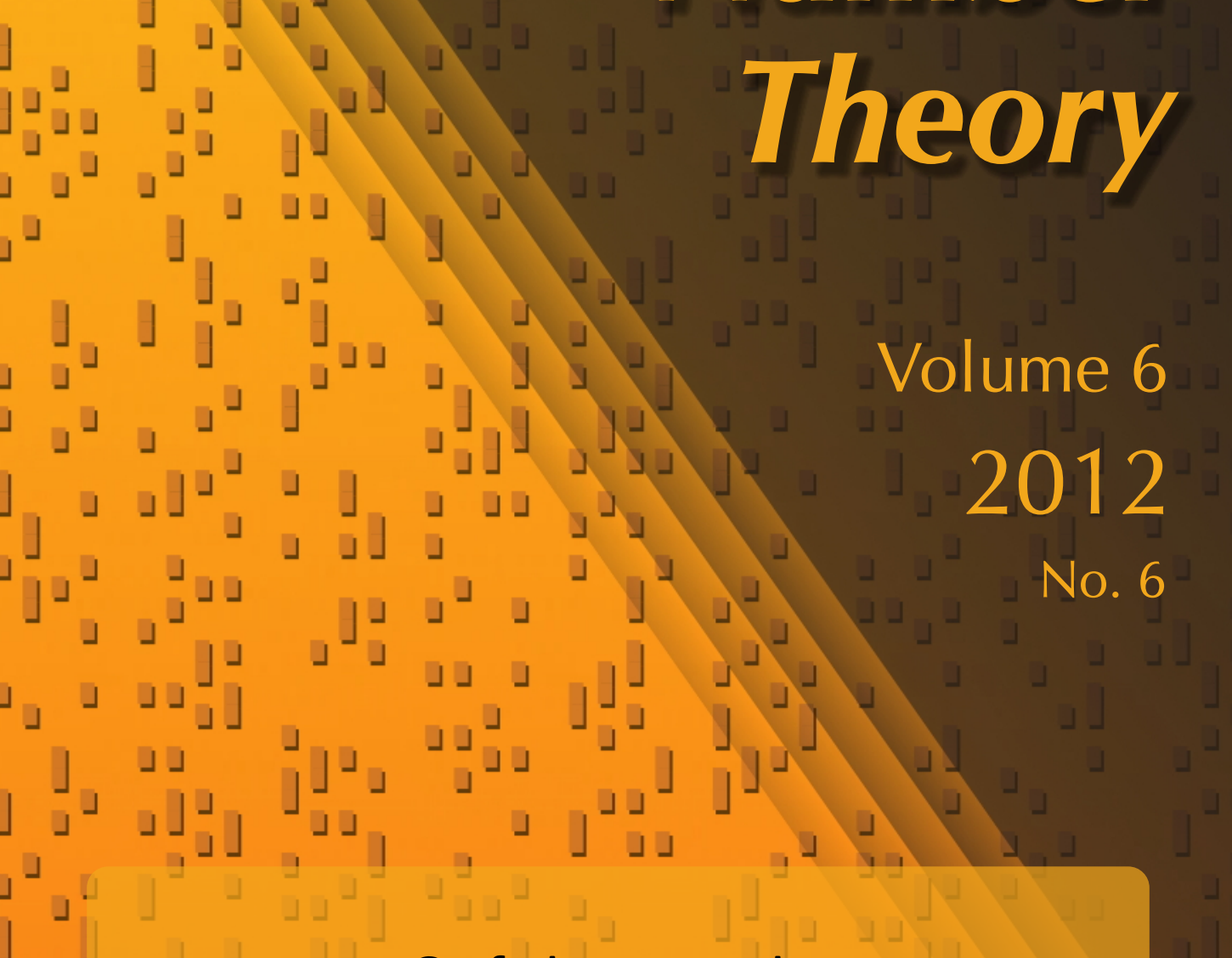

」

\lrcorner

\rfloor

(1)

\lrcorner$\lrcorner\lrcorner$ 


\title{
On fusion categories with few irreducible degrees
}

\author{
Sonia Natale and Julia Yael Plavnik
}

\begin{abstract}
We prove some results on the structure of certain classes of integral fusion categories and semisimple Hopf algebras under restrictions on the set of their irreducible degrees.
\end{abstract}

\section{Introduction}

Let $k$ be an algebraically closed field of characteristic zero. Let $\mathscr{b}$ be a fusion category over $k$. That is, $\mathscr{b}$ is a $k$-linear semisimple rigid tensor category with finitely many isomorphism classes of simple objects, finite-dimensional spaces of morphisms, and such that the unit object 1 of $\mathscr{C}$ is simple.

For example, if $G$ is a finite group, then the categories $\operatorname{Rep} G$ of its finitedimensional representations and the category $\mathscr{b}(G, \omega)$ of $G$-graded vector spaces with associativity determined by the 3-cocycle $\omega$ are fusion categories over $k$. More generally, if $H$ is a finite-dimensional semisimple quasi-Hopf algebra over $k$, then the category $\operatorname{Rep} H$ of its finite-dimensional representations is a fusion category.

Let $\operatorname{Irr}(\mathscr{C})$ denote the set of isomorphism classes of simple objects in the fusion category $\mathscr{C}$. In analogy with the case of finite groups [Isaacs 1976], we shall use the notation c.d.( $\mathscr{C})$ to indicate the set

$$
\text { c.d. }(\mathscr{C})=\{\operatorname{FPdim} x \mid x \in \operatorname{Irr}(\mathscr{C})\} .
$$

Here, FPdim $x$ denotes the Frobenius-Perron dimension of $x \in \operatorname{Irr}(\mathscr{C})$. Notice that, when $\mathscr{C}$ is the representation category of a quasi-Hopf algebra, Frobenius-Perron dimensions coincide with the dimensions of the underlying vector spaces. In this case, we shall use the notation c.d. $(\mathscr{C})=$ c.d. $(H)$.

The positive real numbers $\operatorname{FPdim} x, x \in \operatorname{Irr}(\mathscr{C})$, will be called the irreducible degrees of $\mathscr{b}$.

The fusion categories that we shall consider in this paper are all integral, that is, the Frobenius-Perron dimensions of objects of $\mathscr{C}$ are (natural) integers. By [Etingof

This work was partially supported by CONICET, ANPCyT, and Secyt (UNC).

MSC2010: primary 16T05; secondary 18D10.

Keywords: fusion category, semisimple Hopf algebra, irreducible degree. 
et al. 2005, Theorem 8.33], $\mathscr{C}$ is equivalent to the category of representations of some finite-dimensional semisimple quasi-Hopf algebra.

For a finite group $G$, the knowledge of the set c.d. $(G)=$ c.d. $(k G)$ gives in some cases substantial information about the structure of $G$. It is known, for instance, that if $\mid$ c.d. $(G) \mid \leq 3$, then $G$ is solvable.

On the other hand, if $\mid$ c.d. $(G) \mid=2$, say c.d. $(G)=\{1, m\}, m \geq 1$, then either $G$ has an abelian normal subgroup of index $m$ or else $G$ is nilpotent of class $\leq 3$. Furthermore, if $G$ is nonabelian, then c.d. $(G)=\{1, p\}$ for some prime number $p$, if and only if $G$ contains an abelian normal subgroup of index $p$ or the center $Z(G)$ has index $p^{3}$; see [Isaacs 1976, Theorems 12.11, 12.14, and 12.15].

In the context of semisimple Hopf algebras, some results in the same spirit are known. A basic one is that of [Zhu 1993], which asserts that if $\mid$ c.d. $(H) \mid \leq 3$, then $G\left(H^{*}\right)$ is not trivial; in other words, $H$ has nontrivial characters of degree 1 . A similar result appears in [Natale 1999, Theorem 2.2.3].

Further results, leading to classification theorems in some specific cases, appear in [Izumi and Kosaki 2002] for Kac algebras, that is, Hopf $C^{*}$-algebras.

In this paper we consider the general problem of understanding the structure of a fusion category $\mathscr{C}$ from a knowledge of c.d.( $\mathscr{C})$. For instance, it is well known that c.d. $(\mathscr{C})=\{1\}$ if and only if $\mathscr{C}$ is pointed, if and only if $\mathscr{C} \simeq \mathscr{C}(G, \omega)$, for some 3cocycle $\omega$ on the group $G=G(\mathscr{C})$ of isomorphism classes of invertible objects of $\mathscr{C}$.

More specifically, we address the following question:

Question 1.1. Suppose c.d. $(\mathscr{C})=\{1, p\}$, with $p$ a prime number. What can be said about the structure of $\mathscr{C}$ ?

We treat mostly structural questions regarding nilpotency and solvability, in the sense introduced in [Gelaki and Nikshych 2008] and [Etingof et al. 2011]. (A related question for semisimple Hopf algebras, that we shall not discuss in the present paper, was posed in [Natale 2011, Question 7.2].)

The notions of nilpotency and solvability of a fusion category are related to the corresponding notions for finite groups as follows: if $G$ is a finite group, then the category $\operatorname{Rep} G$ is nilpotent or solvable if and only if $G$ is nilpotent or solvable, respectively. On the dual side, a pointed fusion category $\mathscr{C}(G, \omega)$ is always nilpotent, while it is solvable if and only if the group $G$ is solvable.

An important class of fusion categories, called weakly group-theoretical fusion categories, was introduced and studied in [Etingof et al. 2011]. This generalized in turn the notion of a group-theoretical fusion category of [Etingof et al. 2005]. By definition, $\mathscr{C}$ is group-theoretical if it is Morita equivalent to a pointed fusion category, and it is weakly group-theoretical if it is Morita equivalent to a nilpotent fusion category. Every nilpotent or solvable fusion category is weakly grouptheoretical. 
With regard to Question 1.1, consider, for instance, the case where $\mathscr{C}=\operatorname{Rep} H$, for a semisimple Hopf algebra $H$. A result in this direction is known in the case $p=2$. It is shown in [Bichon and Natale 2011, Corollary 6.6] that if $H$ is a semisimple Hopf algebra such that c.d. $(H) \subseteq\{1,2\}$, then $H$ is upper semisolvable. Moreover, $H$ is necessarily cocommutative if $G\left(H^{*}\right)$ is of order 2. The proof of these results relies on a refinement of [Nichols and Richmond 1996, Theorem 11] given in [Bichon and Natale 2011, Theorem 1.1].

In the context of Kac algebras, it is shown in [Izumi and Kosaki 2002, Theorem IX.8(iii)] that if c.d. $\left(H^{*}\right)=\{1, p\}$ and, in addition, $|G(H)|=p$, then $H$ is a central abelian extension associated to an action of the cyclic group of order $p$ on a nilpotent group. In the recent terminology introduced in [Gelaki and Nikshych 2008], this result implies that such a Kac algebra is nilpotent. See Remark 4.5.

The main results of this paper are summarized in the following theorem.

Theorem 1.2. Let $\mathscr{C}$ be a fusion category over $k$.

(i) (Proposition 7.1) Suppose $\mathfrak{b}$ is weakly group-theoretical and has odd dimension. Then $\mathscr{C}$ is solvable.

Let $p$ be a prime number.

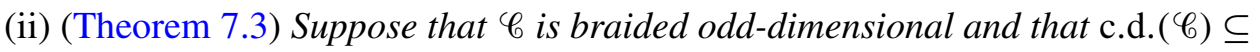
$\left\{p^{m}: m \geq 0\right\}$. Then $\mathscr{C}$ is solvable.

(iii) Suppose c.d. $(\mathscr{C}) \subseteq\{1, p\}$. Then $\mathscr{C}$ is solvable in any of the following cases:

- (Corollary 5.4) $\mathscr{C}$ is of the form $\mathscr{C}\left(G, \omega, \mathbb{Z}_{p}, \alpha\right)$, that is, a group-theoretical fusion category [Etingof et al. 2005], and $G(\mathscr{C})$ is of order $p$.

- (Theorem 6.2) $\mathscr{C}$ is a near-group category [Siehler 2003].

- (Theorem 6.12) $\mathscr{C}=\operatorname{Rep} H$, where $H$ is a semisimple quasitriangular Hopf algebra and $p=2$.

(iv) Let $H$ be a semisimple Hopf algebra such that c.d. $(H) \subseteq\{1, p\}$. Then $H^{*}$ is nilpotent in any of the following cases:

- (Proposition 4.8) $\left|G\left(H^{*}\right)\right|=p$ and $p$ divides $|G(H)|$.

- (Proposition 4.9) $\left|G\left(H^{*}\right)\right|=p$ and $H$ is quasitriangular.

- (Proposition 4.12) H is of type $(1, p ; p, 1)$ as an algebra.

(v) Let $H$ be a semisimple Hopf algebra such that c.d. $(H) \subseteq\{1,2\}$. Then:

- (Theorem 6.4) $H$ is weakly group-theoretical, and, furthermore, it is grouptheoretical if $H=H_{\mathrm{ad}}$.

- (Corollary 6.9) The group $G(H)$ is solvable. 
(vi) (Theorem 4.13) Let $H$ be a semisimple Hopf algebra of type $(1, p ; p, 1)$ as an algebra. Then $H$ is isomorphic to a twisting of the group algebra $k N$, where either $p=2$ and $N=\mathbb{S}_{3}$ or $p=2^{\alpha-1}, \alpha>1$, and $N$ is the affine group of the field $\mathbb{F}_{2^{\alpha}}$.

The proof of part (i) is a consequence of the Feit-Thompson theorem [1963], which asserts that every finite group of odd order is solvable.

By [Natale 2011, Corollary 4.5], the semisimple Hopf algebras $H$ in part (iv) are lower semisolvable in the sense of [Montgomery and Witherspoon 1998].

The results on semisimple Hopf algebras $H$ with c.d. $(H) \subseteq\{1,2\}$ rely on the results of [Bichon and Natale 2011]. We also make strong use of several results of [Gelaki and Nikshych 2008; Gelaki and Naidu 2009; Etingof et al. 2011] on weakly group-theoretical, solvable, and nilpotent fusion categories.

Organization of the paper. In Section 2 we recall the main notions and results relevant to the problem we consider. In particular, several properties of grouptheoretical fusion categories and Hopf algebra extensions are discussed here. The results on nilpotency are contained in Sections 3 and 4. The strategy in these sections consists in reducing the problem to considering Hopf algebra extensions. Sections 5, 6, and 7 are devoted to the solvability question in different situations.

\section{Preliminaries}

2A. Fusion categories. A fusion category over $k$ is a $k$-linear semisimple rigid tensor category $\mathscr{C}$ with finitely many isomorphism classes of simple objects, finitedimensional spaces of morphisms, and such that the unit object 1 of $\mathscr{C}$ is simple. We refer the reader to [Bakalov and Kirillov 2001; Etingof et al. 2005] for basic definitions and facts concerning fusion categories. In particular, if $H$ is a semisimple (quasi-)Hopf algebra over $k$, then $\operatorname{Rep} H$ is a fusion category.

A fusion subcategory of a fusion category $\mathscr{C}$ is a full tensor subcategory $\mathscr{C}^{\prime} \subseteq \mathscr{C}$ such that if $X \in \mathscr{C}$ is isomorphic to a direct summand of an object of ' $\mathscr{C}^{\prime}$, then $X \in \mathscr{C}^{\prime}$. A fusion subcategory is necessarily rigid, so it is indeed a fusion category [Drinfeld et al. 2010, Corollary F.7(i)].

A pointed fusion category is a fusion category where all simple objects are invertible. A pointed fusion category is equivalent to the category $\mathscr{C}(G, \omega)$, of finite-dimensional $G$-graded vector spaces with associativity constraint determined by a cohomology class $\omega \in H^{3}\left(G, k^{\times}\right)$, for some finite group $G$. In other words, $\mathscr{C}(G, \omega)$ is the category of representations of the quasi-Hopf algebra $k^{G}$, with associator $\omega \in\left(k^{G}\right)^{\otimes 3}$.

The fusion subcategory generated by a collection $\mathscr{L}$ of objects of $\mathscr{C}$ is the smallest fusion subcategory containing $\mathscr{X}$.

If $\mathscr{C}$ is a fusion category, then the set of isomorphism classes of invertible objects of $\mathscr{C}$ forms a group, denoted $G(\mathscr{C})$. The fusion subcategory generated by the 
invertible objects of $\mathscr{C}$ is a fusion subcategory, denoted $\mathscr{C}_{\mathrm{pt}}$; it is the maximal pointed subcategory of $\mathscr{C}$.

Let $\operatorname{Irr}(\mathscr{C})$ denote the set of isomorphism classes of simple objects in the fusion category $\mathscr{C}$. The set $\operatorname{Irr}(\mathscr{C})$ is a basis over $\mathbb{Z}$ of the Grothendieck ring $\mathscr{G}(\mathscr{C})$.

2B. Irreducible degrees. For $x \in \operatorname{Irr}(\mathscr{C})$, let FPdim $x$ be its Frobenius-Perron dimension. The positive real numbers FPdim $x, x \in \operatorname{Irr}(\mathscr{C})$, will be called the irreducible degrees of $\mathscr{C}$. These extend to a ring homomorphism FPdim: $\mathscr{G}(\mathscr{C}) \rightarrow \mathbb{R}$. When $\mathscr{b}$ is the representation category of a quasi-Hopf algebra, Frobenius-Perron dimensions coincide with the dimensions of the underlying vector spaces.

The set of irreducible degrees of $\mathscr{C}$ is defined as

$$
\text { c.d. }(\mathscr{C})=\{\operatorname{FPdim} x \mid x \in \operatorname{Irr}(\mathscr{C})\} \text {. }
$$

The category $\mathscr{C}$ is called integral if c.d. $(\mathscr{C}) \subseteq \mathbb{N}$.

If $X$ is any object of $\mathscr{C}$, then its class $x$ in $\mathscr{G}(\mathscr{C})$ decomposes as

$$
x=\sum_{y \in \operatorname{Irr}(\mathscr{C})} m(y, x) y,
$$

where $m(y, x)=\operatorname{dim} \operatorname{Hom}(Y, X)$ is the multiplicity of $Y$ in $X$, if $Y$ is an object representing the class $y \in \operatorname{Irr}(\mathscr{C})$.

For all $x, y, z \in \mathscr{G}(\mathscr{C})$, we have:

$$
m(x, y z)=m\left(y^{*}, z x^{*}\right)=m\left(y, x z^{*}\right) .
$$

Let $x \in \operatorname{Irr}(\mathscr{C})$. The stabilizer of $x$ under left multiplication by elements of $G(\mathscr{C})$ in the Grothendieck ring will be denoted by $G[x]$. So, an invertible element $g \in G(\mathscr{C})$ belongs to $G[x]$ if and only if $g x=x$.

In view of (2-1), for all $x \in \operatorname{Irr}(\mathscr{C})$, we have

$$
G[x]=\left\{g \in G(\mathscr{C}): m\left(g, x x^{*}\right)>0\right\}=\left\{g \in G(\mathscr{C}): m\left(g, x x^{*}\right)=1\right\} .
$$

In particular, we have the following relation in $\mathscr{G}(\mathscr{C})$ :

$$
x x^{*}=\sum_{g \in G[x]} g+\sum_{\substack{y \in \operatorname{Irr}(\mathscr{C}) \\ \operatorname{FPdim} y>1}} m\left(y, x x^{*}\right) y .
$$

Remark 2.1. An object $g \in \mathscr{C}$ is invertible if and only if FPdim $g=1$.

Suppose that $\mathscr{C}$ is an integral fusion category with $\mid$ c.d. $(\mathscr{C}) \mid=2$. That is, c.d. $(\mathscr{C})=\{1, d\}$ for some integer $d>1$. We claim that $d$ divides the order of $G[x]$ for all $x \in \operatorname{Irr}(\mathscr{C})$ with FPdim $x>1$; in particular, $d$ divides the order of $G(\mathscr{C})$, and thus $G(\mathscr{C}) \neq 1$. 
Indeed, if $x \in \operatorname{Irr}(\mathscr{C})$ with FPdim $x=d$, we have the relation

$$
x x^{*}=\sum_{g \in G[x]} g+\sum_{\substack{y \in \operatorname{Irr}(\mathscr{C}) \\ \text { FPdim } y=d}} m\left(y, x x^{*}\right) y .
$$

The claim follows by taking Frobenius-Perron dimensions.

2C. Semisimple Hopf algebras. Let $H$ be a semisimple Hopf algebra over $k$. We next recall some of the terminology and conventions from [Natale 2007b] that will be used throughout this paper.

As an algebra, $H$ is isomorphic to a direct sum of full matrix algebras

$$
H \simeq k^{(n)} \oplus \bigoplus_{i=1}^{r} M_{d_{i}}(k)^{\left(n_{i}\right)},
$$

where $n=\left|G\left(H^{*}\right)\right|$. The Nichols-Zoeller theorem [Nichols and Zoeller 1989] implies that $n$ divides both $\operatorname{dim} H$ and $n_{i} d_{i}^{2}$, for all $i=1, \ldots, r$.

If we have an isomorphism as in (2-2), we shall say that $H$ is of type $\left(1, n ; d_{1}, n_{1}\right.$; $\left.\ldots ; d_{r}, n_{r}\right)$ as an algebra. If $H^{*}$ is of type $\left(1, n ; d_{1}, n_{1} ; \ldots ; d_{r}, n_{r}\right)$ as an algebra, we shall say that $H$ is of type $\left(1, n ; d_{1}, n_{1} ; \ldots ; d_{r}, n_{r}\right)$ as a coalgebra.

Let $V$ be an $H$-module. The character of $V$ is the element $\chi=\chi_{V} \in H^{*}$ defined by $\chi(h)=\operatorname{Tr}_{V}(h)$, for all $h \in H$. For a character $\chi$, its degree is the integer $\operatorname{deg} \chi=\chi(1)=\operatorname{dim} V$. The character $\chi_{V}$ is called irreducible if $V$ is irreducible.

The set $\operatorname{Irr}(H)$ of irreducible characters of $H$ spans a semisimple subalgebra $R(H)$ of $H^{*}$, called the character algebra of $H$. It is isomorphic, under the map $V \rightarrow \chi_{V}$, to the extension of scalars $k \otimes_{\mathbb{Z}} \mathscr{G}(\operatorname{Rep} H)$ of the Grothendieck ring of the category $\operatorname{Rep} H$. In particular, there is an identification $\operatorname{Irr}(H) \simeq \operatorname{Irr}(\operatorname{Rep} H)$.

Under this identification, all properties listed in Section 2B hold true for characters.

In this context, we have $G(\operatorname{Rep} H)=G\left(H^{*}\right)$. The stabilizer of $\chi$ under left multiplication by elements in $G\left(H^{*}\right)$ will be denoted by $G[\chi]$. By the NicholsZoeller theorem [Nichols and Zoeller 1989], we have that $|G[\chi]|$ divides $(\operatorname{deg} \chi)^{2}$.

Following [Isaacs 1976, Chapter 12], we use the notation c.d. $(H)=$ c.d. $(\operatorname{Rep} H)$. Hence,

$$
\text { c.d. }(H)=\{\operatorname{deg} \chi \mid \chi \in \operatorname{Irr}(H)\} .
$$

In particular, if $H$ is of type $\left(1, n ; d_{1}, n_{1} ; \ldots ; d_{r}, n_{r}\right)$ as an algebra, then c.d. $(H)=$ $\left\{1, d_{1}, \ldots, d_{r}\right\}$.

There is a bijective correspondence between Hopf algebra quotients of $H$ and standard subalgebras of $R(H)$, that is, subalgebras spanned by irreducible characters of $H$. This correspondence assigns to the Hopf algebra quotient $H \rightarrow \bar{H}$ its character algebra $R(\bar{H}) \subseteq R(H)$. See [Nichols and Richmond 1996]. 
2D. Group-theoretical categories. A group-theoretical fusion category is a fusion category Morita equivalent to a pointed fusion category $\mathscr{C}(G, \omega)$. Such a fusion category is equivalent to the category $\mathscr{C}(G, \omega, F, \alpha)$ of $k_{\alpha} F$-bimodules in $\mathscr{C}(G, \omega)$, where $G$ is a finite group, $\omega$ is a 3-cocycle on $G, F \subseteq G$ is a subgroup, and $\alpha \in C^{2}\left(F, k^{\times}\right)$is a 2-cochain on $F$ such that $\left.\omega\right|_{F}=d \alpha$. A semisimple Hopf algebra $H$ is called group-theoretical if the category Rep $H$ is group-theoretical.

Let $\mathscr{C}=\mathscr{C}(G, \omega, F, \alpha)$ be a group-theoretical fusion category. Let also $\Gamma$ be a subgroup of $G$, endowed with a 2-cocycle $\beta \in Z^{2}\left(\Gamma, k^{\times}\right)$, such that:

- The class $\left.\omega\right|_{\Gamma}$ is trivial.

- $G=F \Gamma$.

- The class $\left.\left.\alpha\right|_{F \cap \Gamma} \beta^{-1}\right|_{F \cap \Gamma}$ is nondegenerate.

Then there is an associated semisimple Hopf algebra $H$, such that the category Rep $H$ is equivalent to $\mathscr{C}$. By [Ostrik 2003], equivalence classes of subgroups $\Gamma$ of $G$ satisfying the conditions above classify fiber functors $\mathscr{C} \mapsto$ Vec; these correspond to the distinct Hopf algebras $H$.

Let $\mathscr{C}=\mathscr{C}(G, \omega, F, \alpha)$ be a group-theoretical fusion category. The simple objects of $\mathscr{C}$ are classified by pairs $\left(s, U_{s}\right)$, where $s$ runs over a set of representatives of the double cosets of $F$ in $G$, that is, orbits of the action of $F$ in the space $F \backslash G$ of left cosets of $F$ in $G, F_{s}=F \cap s F s^{-1}$ is the stabilizer of $s \in F \backslash G$, and $U_{s}$ is an irreducible representation of the twisted group algebra $k_{\sigma_{s}} F_{s}$, that is, an irreducible projective representation of $F_{s}$ with respect to a certain 2-cocycle $\sigma_{s}$ determined by $\omega$; see [Gelaki and Naidu 2009, Theorem 5.1].

The irreducible representation $W_{\left(s, U_{s}\right)}$ corresponding to such a pair $\left(s, U_{s}\right)$ has dimension

$$
\operatorname{dim} W_{\left(s, U_{s}\right)}=\left[F: F_{s}\right] \operatorname{dim} U_{s} .
$$

Corollary 2.2. The irreducible degrees of $\mathscr{C}(G, \omega, F, \alpha)$ divide the order of $F$.

Remark 2.3. A group-theoretical category $\mathscr{b}=\mathscr{b}(G, \omega, F, \alpha)$ is an integral fusion category. An explicit construction of a quasi-Hopf algebra $H$ such that $\operatorname{Rep} H \simeq \mathscr{C}$ was given in [Natale 2005].

As an algebra, $H$ is a crossed product $k^{F \backslash G} \#_{\sigma} k F$, where $F \backslash G$ is the space of left cosets of $F$ in $G$ with the natural action of $F$, and $\sigma$ is a certain 2-cocycle determined by $\omega$.

Irreducible representations of $H$, that is, simple objects of $\mathscr{C}$, can therefore be described using the results for group crossed products in [Montgomery and Witherspoon 1998]: this is done in [Natale 2005, Proposition 5.5].

By [Gelaki and Naidu 2009, Theorem 5.2], the group $G(\mathscr{C})$ of invertible objects of $\mathscr{C}$ fits into an exact sequence

$$
1 \rightarrow \widehat{F} \rightarrow G(\mathscr{C}) \rightarrow K \rightarrow 1,
$$


where $K=\left\{x \in N_{G}(F):\left[\sigma_{x}\right]=1\right\}$.

2E. Abelian extensions. Suppose that $G=F \Gamma$ is an exact factorization of the finite group $G$, where $\Gamma$ and $F$ are subgroups of $G$. Equivalently, $F$ and $\Gamma$ form a matched pair of groups with the actions $\triangleleft: \Gamma \times F \rightarrow \Gamma$ and $\triangleright: \Gamma \times F \rightarrow F$, defined by $s x=(x \triangleleft s)(x \triangleright s), x \in F, s \in \Gamma$. In this case, $G$ is isomorphic to the group $F \bowtie \Gamma$ defined as follows: $F \bowtie \Gamma=F \times \Gamma$, with multiplication $(x, s)(t, y)=(x(s \triangleright y),(s \triangleleft y) t)$, for all $x, y \in F, s, t \in \Gamma$.

Let $\sigma \in Z^{2}\left(F,\left(k^{\Gamma}\right)^{\times}\right)$and $\tau \in Z^{2}\left(\Gamma,\left(k^{F}\right)^{\times}\right)$be normalized 2-cocycles with respect to the actions afforded, respectively, by $\triangleleft$ and $\triangleright$, subject to appropriate compatibility conditions [Masuoka 1999].

The bicrossed product $H=k^{\Gamma \tau} \#_{\sigma} k F$ associated to this data is a semisimple Hopf algebra. There is an abelian exact sequence

$$
k \rightarrow k^{\Gamma} \rightarrow H \rightarrow k F \rightarrow k .
$$

Moreover, every Hopf algebra $H$ fitting into such an exact sequence can be described in this way. This gives a bijective correspondence between the equivalence classes of Hopf algebra extensions (2-5) associated to the matched pair $(F, \Gamma)$ and a certain abelian group $\operatorname{Opext}\left(k^{\Gamma}, k F\right)$.

Remark 2.4. The Hopf algebra $H$ is group theoretical. In fact, by [Natale 2003, Section 4.2], we have an equivalence of fusion categories $\operatorname{Rep} H \simeq \mathscr{C}(G, \omega, F, 1)$, where $\omega$ is the 3-cocycle on $G$ coming from the so-called Kac exact sequence.

Irreducible representations of $H$ are classified by pairs $\left(s, U_{s}\right)$, where $s$ runs over a set of representatives of the orbits of the action of $F$ in $\Gamma, F_{s}=F \cap s F s^{-1}$ is the stabilizer of $s \in \Gamma$, and $U_{s}$ is an irreducible representation of the twisted group algebra $k_{\sigma_{s}} F_{s}$, that is, an irreducible projective representation of $F_{s}$ with cocycle $\sigma_{s}$, where $\sigma_{s}(x, y)=\sigma(x, y)(s), x, y \in F, s \in \Gamma$; see [Kashina et al. 2002].

Note that, for all $s \in \Gamma$, the restriction of $\sigma_{s}: F \times F \rightarrow k^{\times}$to the stabilizer $F_{s}$ indeed defines a 2-cocycle on $F_{s}$.

The irreducible representation corresponding to such a pair $\left(s, U_{s}\right)$ is in this case of the form

$$
W_{\left(s, U_{s}\right)}:=\operatorname{Ind}_{k^{\Gamma} \otimes k F_{s}}^{H} s \otimes U_{s} .
$$

2F. Quasitriangular Hopf algebras. Let $H$ be a finite-dimensional Hopf algebra. Recall that $H$ is called quasitriangular if there exists an invertible element $R \in$ $H \otimes H$, called an $R$-matrix, such that

$$
\begin{array}{ll}
(\Delta \otimes \mathrm{id})(R)=R_{13} R_{23}, & (\epsilon \otimes \mathrm{id})(R)=1, \\
(\mathrm{id} \otimes \Delta)(R)=R_{13} R_{12}, & (\mathrm{id} \otimes \epsilon)(R)=1, \\
\Delta^{\mathrm{cop}}(h)=R \Delta(h) R^{-1}, & \text { for all } h \in H .
\end{array}
$$


The existence of an $R$-matrix (also called a quasitriangular structure in what follows) amounts to the category $\operatorname{Rep} H$ being a braided tensor category; see [Bakalov and Kirillov 2001].

For instance, the group algebra $k G$ of a finite group $G$ is a quasitriangular Hopf algebra with $R=1 \otimes 1$. On the other hand, the dual Hopf algebra $k^{G}$ admits a quasitriangular structure if and only if $G$ is abelian.

If it exists, a quasitriangular structure in a Hopf algebra $H$ need not be unique.

Another example of a quasitriangular Hopf algebra is the Drinfeld double $D(H)$ of $H$, where $H$ is any finite-dimensional Hopf algebra. We have $D(H)=H^{* \operatorname{cop}} \otimes H$ as coalgebras, with a canonical $R$-matrix $\mathscr{R}=\sum_{i} h^{i} \otimes h_{i}$, where $\left(h_{i}\right)_{i}$ is a basis of $H$ and $\left(h^{i}\right)_{i}$ is the dual basis.

As braided tensor categories, $\operatorname{Rep} D(H)=\mathscr{L}(\operatorname{Rep} H)$ is equivalent to the center of the tensor category Rep $H$.

Suppose $(H, R)$ is a quasitriangular Hopf algebra. There are Hopf algebra maps $f_{R}: H^{* \text { cop }} \rightarrow H$ and $f_{R_{21}}: H^{*} \rightarrow H^{\text {op }}$ defined by

$$
f_{R}(p)=p\left(R^{(1)}\right) R^{(2)}, \quad f_{R_{21}}(p)=p\left(R^{(2)}\right) R^{(1)},
$$

for all $p \in H^{*}$, where $R=R^{(1)} \otimes R^{(2)} \in H \otimes H$.

We shall denote $f_{R}\left(H^{*}\right)=H_{+}$and $f_{R_{21}}\left(H^{*}\right)=H_{-}$, respectively. Hence $H_{+}$ and $H_{-}$are Hopf subalgebras of $H$ and we have $H_{+} \simeq\left(H_{-}^{*}\right)^{\text {cop }}$.

We shall also denote by $H_{R}=H_{-} H_{+}=H_{+} H_{-}$the minimal quasitriangular Hopf subalgebra of $H$; see [Radford 1993].

By [Radford 1993, Theorem 2], the multiplication of $H$ determines a surjective Hopf algebra map $D\left(H_{-}\right) \rightarrow H_{R}$.

A quasitriangular Hopf algebra $(H, R)$ is called factorizable if the map $\Phi_{R}$ : $H^{*} \rightarrow H$ is an isomorphism, where

$$
\Phi_{R}(p)=p\left(Q^{(1)}\right) Q^{(2)}, \quad p \in H^{*}
$$

here, $Q=Q^{(1)} \otimes Q^{(2)}=R_{21} R \in H \otimes H$ [Reshetikhin and Semenov-Tian-Shansky 1988].

If on the other hand $\Phi_{R}=\epsilon 1$ (or equivalently, $R_{21} R=1 \otimes 1$ ), then $(H, R)$ is called triangular. Finite-dimensional triangular Hopf algebras were completely classified in [Etingof and Gelaki 2003]. In particular, if $(H, R)$ is a semisimple quasitriangular Hopf algebra, then $H$ is isomorphic, as a Hopf algebra, to a twisting $(k G)^{J}$ of some finite group $G$.

It is well known that the Drinfeld double $(D(H), \mathscr{R})$ is indeed a factorizable quasitriangular Hopf algebra. We have $D(H)_{+}=H$ and $D(H)_{-}=H^{* \text { cop }}$.

We shall use later on in this paper the following result about factorizable Hopf algebras. A categorical version is established in [Gelaki and Nikshych 2008]. 
Theorem 2.5 [Schneider 2001, Theorem 2.3]. Let $(H, R)$ be a factorizable Hopf algebra. Then the map $\Phi_{R}$ induces an isomorphism of groups $G\left(H^{*}\right) \rightarrow G(H) \cap$ $Z(H)$.

Note that we may identify $G(D(H))=G\left(H^{*}\right) \times G(H)$. Under this identification, Theorem 2.5 gives us a group isomorphism

$$
G\left(D(H)^{*}\right) \rightarrow G(D(H)) \cap Z(D(H)),
$$

such that $g \# f \mapsto f \# g$. See also [Radford 1993].

In particular, if $f=\epsilon$, then $g \in G(H) \cap Z(H)$, and also if $g=1$, then $f \in$ $G\left(H^{*}\right) \cap Z\left(H^{*}\right)$.

Suppose $(H, R)$ is a finite-dimensional quasitriangular Hopf algebra, and let $D(H)$ be the Drinfeld double of $H$. Then there is a surjective Hopf algebra map $f: D(H) \rightarrow H$, such that $(f \otimes f) \mathscr{R}=R$. The map $f$ is determined by $f(p \otimes h)=f_{R}(p) h$, for all $p \in H^{*}, h \in H$.

This corresponds to the canonical inclusion of the braided tensor category Rep $H$ (with braiding determined by the action of the $R$-matrix) into its center.

In particular, in the case where $H$ is quasitriangular, the group $G\left(H^{*}\right)$ can be identified with a subgroup of $G\left(D(H)^{*}\right)$.

\section{Nilpotency}

Let $G$ be a finite group. A $G$-grading of a fusion category $\mathscr{C}$ is a decomposition of $\mathscr{C}$ as a direct sum of full abelian subcategories $\mathscr{C}=\bigoplus_{g \in G} \mathscr{b}_{g}$, such that $\mathscr{C}_{g}^{*}=\mathscr{b}_{g-1}$ and the tensor product $\otimes: \mathscr{C} \times \mathscr{C} \rightarrow \mathscr{C}$ maps $\mathscr{C}_{g} \times \mathscr{C}_{h}$ to $\mathscr{C}_{g h}$. The neutral component $\mathscr{C}_{e}$ is thus a fusion subcategory of $\mathscr{C}$.

The grading is called faithful if $\mathscr{C}_{g} \neq 0$, for all $g \in G$. In this case, $\mathscr{C}$ is called a $G$-extension of $\mathscr{C}_{e}$ [Etingof et al. 2011].

The following proposition is a consequence of [Gelaki and Nikshych 2008, Theorem 3.8].

Proposition 3.1. Let $\mathscr{C}=\operatorname{Rep} H$, where $H$ is a semisimple Hopf algebra. Then a faithful $G$-grading on $\mathscr{C}$ corresponds to a central exact sequence of Hopf algebras $k \rightarrow k^{G} \rightarrow H \rightarrow \bar{H} \rightarrow k$, such that $\operatorname{Rep} \bar{H}=\mathscr{C}_{e}$.

Let $\mathscr{C}$ be a fusion category and let $\mathscr{C}_{\text {ad }}$ be the adjoint subcategory of $\mathscr{C}$. That is, $\mathscr{C}_{\text {ad }}$ is the fusion subcategory of $\mathscr{C}$ generated by $X \otimes X^{*}$, where $X$ runs through the simple objects of $\mathscr{C}$.

It is shown in [Gelaki and Nikshych 2008] that there is a canonical faithful grading on $\mathscr{C}: \mathscr{C}=\bigoplus_{g \in U(\mathscr{C})} \mathscr{b}_{g}$, called the universal grading, such that $\mathscr{C}_{e}=\mathscr{C}_{\text {ad }}$. The group $U(\mathscr{C})$ is called the universal grading group of $\mathscr{b}$. 
In the case where $\mathscr{C}=\operatorname{Rep} H$, for a semisimple Hopf algebra $H, K=k^{U(\mathscr{C})}$ is the maximal central Hopf subalgebra of $H$ and $\mathscr{C}_{\mathrm{ad}}=\operatorname{Rep} H / H K^{+}$[Gelaki and Nikshych 2008, Theorem 3.8].

Recall from [Gelaki and Nikshych 2008; Etingof et al. 2011] that a fusion category $\mathscr{b}$ is called (cyclically) nilpotent if there is a sequence of fusion categories

$$
\mathscr{C}_{0}=\mathrm{Vec}, \mathscr{C}_{1}, \ldots, \mathscr{C}_{n}=\mathscr{C}
$$

and a sequence $G_{1}, \ldots, G_{n}$ of finite (cyclic) groups such that $\mathscr{C}_{i}$ is faithfully graded by $G_{i}$ with trivial component $\mathscr{C}_{i-1}$.

The semisimple Hopf algebra $H$ is called nilpotent if the fusion category Rep $H$ is nilpotent [Gelaki and Nikshych 2008, Definition 4.4].

For instance, if $G$ is a finite group, then the dual group algebra $k^{G}$ is always nilpotent. However, the group algebra $k G$ is nilpotent if and only if the group $G$ is nilpotent [Gelaki and Nikshych 2008, Remark 4.7(1)].

3A. Nilpotency of an abelian extension. It is shown in [Gelaki and Naidu 2009, Corollary 4.3] that a group-theoretical fusion category $\mathscr{C}(G, \omega, F, \alpha)$ is nilpotent if and only if the normal closure of $F$ in $G$ is nilpotent. On the other hand, this happens if and only if $F$ is nilpotent and subnormal in $G$, if and only if $F \subseteq \operatorname{Fit}(G)$, where $\operatorname{Fit}(G)$ is the Fitting subgroup of $G$, that is, the unique largest normal nilpotent subgroup of $G$ [Gelaki and Naidu 2009, §2.3].

Combined with Remark 2.4, this implies:

Proposition 3.2. Let $k \rightarrow k^{\Gamma} \rightarrow H \rightarrow k F \rightarrow k$ be an abelian exact sequence and let $G=F \bowtie \Gamma$ be the associated factorizable group. Then $H$ is nilpotent if and only if $F \subseteq \operatorname{Fit}(G)$.

An abelian exact sequence (2-5) is called central if the image of $k^{\Gamma}$ is a central Hopf subalgebra of $H$. It is called cocentral if the dual exact sequence is central.

The following facts are well known:

Lemma 3.3. Consider an abelian exact sequence (2-5).

(i) The sequence is central if and only if the action $\triangleleft: \Gamma \times F \rightarrow \Gamma$ is trivial. In this case, the group $G=F \bowtie \Gamma$ is a semidirect product $G \simeq F \rtimes \Gamma$ with respect to the action $\triangleright: \Gamma \times F \rightarrow F$.

(ii) The sequence is cocentral if and only if the action $\triangleright: \Gamma \times F \rightarrow F$ is trivial. In this case, the group $G=F \bowtie \Gamma$ is a semidirect product $G \simeq F \ltimes \Gamma$ with respect to the action $\triangleleft: \Gamma \times F \rightarrow \Gamma$.

Remark 3.4. Assume the exact sequence (2-5) is central. Then $F$ is a normal subgroup of $G$. It follows from Proposition 3.2 that $H$ is nilpotent if and only if $F$ is nilpotent. 


\section{On the nilpotency of a class of semisimple Hopf algebras}

Let $p$ be a prime number. We shall consider in this subsection a nontrivial semisimple Hopf algebra $H$ fitting into an abelian exact sequence

$$
k \rightarrow k^{\mathbb{Z}_{p}} \rightarrow H \rightarrow k F \rightarrow k .
$$

The main result of this subsection is Proposition 4.3 below.

Suppose that $\mathscr{b}$ is any group-theoretical fusion category of the form $\mathscr{b}=$ $\mathscr{C}\left(G, \omega, \mathbb{Z}_{p}, \alpha\right)$ (note that we may assume that $\alpha=1$ ). In particular, $p$ divides the order of $G(\mathscr{C})$. We also have c.d. $(\mathscr{C}) \subseteq\{1, p\}$, by Corollary 2.2.

Lemma 4.1. Let $\mathscr{C}=\mathscr{C}\left(G, \omega, \mathbb{Z}_{p}, \alpha\right)$. Assume that $|G(\mathscr{C})|=p$. Then $G$ is a Frobenius group with Frobenius complement $\mathbb{Z}_{p}$.

Proof. The description of the irreducible representations of $\mathscr{b}$ in Section 2D, combined with the assumption that $|G(\mathscr{C})|=p$, implies that $g \mathbb{Z}_{p} g^{-1} \cap \mathbb{Z}_{p}=\{e\}$, for all $g \in G \backslash \mathbb{Z}_{p}$. (In particular, the action of $\mathbb{Z}_{p}$ on $\mathbb{Z}_{p} \backslash G$ has no fixed points $s \neq e$.)

This condition says that $G$ is a Frobenius group with Frobenius complement $\mathbb{Z}_{p}$, as claimed.

Remark 4.2. Let $G$ be a Frobenius group with Frobenius complement $\mathbb{Z}_{p}$, as in Lemma 4.1. By the Frobenius theorem we have that the Frobenius kernel $N$ is a normal subgroup of $G$, such that $G$ is a semidirect product $G=N \rtimes \mathbb{Z}_{p}$. Moreover, $N$ is a nilpotent group, by a theorem of Thompson. See [Isaacs 1976, Theorem 7.2; Robinson 1982, Theorem 10.5.6]. In fact, the Frobenius kernel $N$ is equal to $\operatorname{Fit}(G)$, the Fitting subgroup of $G$ [Robinson 1982, Exercise 10.5.8].

As a consequence we get the following:

Proposition 4.3. Consider the abelian exact sequence (4-1) and assume that $|G(H)|=p$.

(i) The sequence is central, that is, $G(H) \subseteq Z(H)$.

(ii) $G=F \bowtie \mathbb{Z}_{p}$ is a Frobenius group with kernel $F$. In particular, $F$ is nilpotent.

Proof. We follow the lines of the proof of [Izumi and Kosaki 2002, Proposition X.7(i)]. Consider the matched pair $\left(F, \mathbb{Z}_{p}\right)$ associated to (4-1), as in Section 2E. Let $G=F \bowtie \mathbb{Z}_{p}$ be the corresponding factorizable group.

We have an equivalence of fusion categories $\operatorname{Rep} H^{*} \simeq \mathscr{C}\left(G, \omega, \mathbb{Z}_{p}, 1\right)$; see Remark 2.4. Then $\operatorname{Rep} H^{*}$ is group-theoretical and, by assumption, $G\left(\operatorname{Rep} H^{*}\right)$ is of order $p$. By Lemma 4.1, $G$ is a Frobenius group with Frobenius complement $\mathbb{Z}_{p}$. Therefore $G$ is a semidirect product $G=N \rtimes \mathbb{Z}_{p}$, where $N=\operatorname{Fit}(G)$ is a nilpotent subgroup (see Remark 4.2). 
Since $|G(H)|=p$, then the action of $\mathbb{Z}_{p}$ on $F$ has no fixed points. It follows, after decomposing $F$ as a disjoint union of $\mathbb{Z}_{p}$-orbits, that $|F|=1(\bmod p)$. In particular, $|F|$ is not divisible by $p$. Then $F$ must map trivially under the canonical projection $G \rightarrow G / N$, that is, $F \subseteq N$. Hence $F=N$, because they have the same order. This shows (ii). Since $F$ is normal in $G$, we get (i) in view of Lemma 3.3.

Corollary 4.4. Let $k \rightarrow k^{\mathbb{Z}_{p}} \rightarrow H \rightarrow k F \rightarrow k$ be an abelian exact sequence such that $|G(H)|=p$. Then $H$ is nilpotent.

Proof. It follows from Proposition 4.3, in view of Remark 3.4.

Remark 4.5. In view of [Izumi and Kosaki 2002, Theorem IX.8(iii)], if $H$ is a Kac algebra with c.d. $\left(H^{*}\right)=\{1, p\}$ and $|G(H)|=p$, then $H$ is a central abelian extension associated to an action of the cyclic group of order $p$ on a nilpotent group. It follows from Corollary 4.4 that $H$ is a nilpotent Hopf algebra.

Remark 4.6. Note that the (dual) assumption that c.d. $(H)=\{1, p\}$ does not imply that $H$ is nilpotent in general. For example, take $H$ to be the group algebra of a nonabelian semidirect product $F \rtimes \mathbb{Z}_{p}$, where $F$ is an abelian group such that $(|F|, p)=1$.

On the other hand, the assumption on $|G(H)|$ in Corollary 4.4 and Proposition 4.3 is essential. Namely, for all prime number $p$, there exist semisimple Hopf algebras $H$ with c.d. $\left(H^{*}\right)=\{1, p\}$ and such that $H$ is not nilpotent.

To see an example, consider a group $F$ with an automorphism of order $p$ and suppose $F$ is not nilpotent (take, for instance, $F=\mathbb{S}_{n}$, a symmetric group, such that $n>6$ is sufficiently large). Consider the corresponding action of $\mathbb{Z}_{p}$ on $F$ by group automorphisms and let $G=F \rtimes \mathbb{Z}_{p}$ be the semidirect product.

Then there is an associated (split) abelian exact sequence $k \rightarrow k^{\mathbb{Z}_{p}} \rightarrow H \rightarrow$ $k F \rightarrow k$, such that $H$ is not commutative and not cocommutative. Moreover, in view of Corollary 2.2, c.d. $\left(H^{*}\right)=\{1, p\}$. But, by Remark 3.4, $H$ is not nilpotent, because $F$ is not nilpotent by assumption.

4A. Reduction to abelian extensions from character degrees. In this subsection we consider the case where c.d. $(H)=\{1, p\}$ for some prime $p$ and $\left|G\left(H^{*}\right)\right|=p$. We treat the problem of deducing an abelian extension like (4-1) from this assumption.

It is known, for instance, that if $p=2$, then the assumption implies that $H$ is cocommutative [Izumi and Kosaki 2002, Corollary IX.9; Bichon and Natale 2011, Proposition 6.8].

Lemma 4.7. If c.d. $\left(H^{*}\right)=\{1, p\}$ for some prime $p$, then $H /(k G(H))^{+} H$ is a cocommutative coalgebra.

Proof. Let $\chi$ be an irreducible character of degree $p$. We have that

$$
\chi \chi^{*}=\sum_{g \in G[\chi]} g+\sum_{\operatorname{deg} \lambda=p} \lambda .
$$


So $p|| G[\chi] \mid$. Therefore $|G[\chi]|$ is either $p=\operatorname{deg} \chi$ or $p^{2}$, because it divides $(\operatorname{deg} \chi)^{2}$.

Moreover, since $\chi=g \chi$ for all $g \in G[\chi]$, we have $G[\chi] C=C$, where $C$ is the simple subcoalgebra of $H$ containing $\chi$. Then it follows from [Natale 2007b, Remark 3.2.7] that $C /(k G[\chi])^{+} C$ is a cocommutative coalgebra (indeed, $|G[\chi]|$ is either $p=\operatorname{deg} \chi$ or $p^{2}$, but in the last case, $C /(k G[\chi])^{+} C$ is one-dimensional, hence also cocommutative). Then $H /(k G(H))^{+} H$ is a cocommutative coalgebra, by [Natale 2007b, Corollary 3.3.2].

4B. Results for the type $(1, p ; p, n)$. Let $p$ be a prime number. In this subsection $H$ will be a semisimple Hopf algebra such that c.d. $(H)=\{1, p\}$ and $\left|G\left(H^{*}\right)\right|=p$. Hence $H$ is of type $(1, p ; p, n)$ as an algebra.

Proposition 4.8. Suppose that $p$ divides $|G(H)|$. Then $G\left(H^{*}\right) \subseteq Z\left(H^{*}\right)$ and $H^{*}$ is nilpotent.

Proof. By assumption, there is a subgroup $G$ of $G(H)$ with $|G|=p$ (that is, $G \simeq \mathbb{Z}_{p}$ ) and the Hopf algebra inclusion $k G \rightarrow H$ induces the following sequence:

$$
k G\left(H^{*}\right) \stackrel{i}{\rightarrow} H^{*} \stackrel{\pi}{\rightarrow} k G
$$

with $\pi$ surjective. Set $A=k G\left(H^{*}\right)$ and $B=k G$. By [Natale 2007b, Lemma 4.1.9], $\pi \circ i: k G\left(H^{*}\right) \rightarrow k G$ is an isomorphism and $H^{*} \simeq R \# k G\left(H^{*}\right) \simeq R \# \mathbb{Z}_{p}$ is a biproduct, where $R \doteq\left(H^{*}\right)^{\text {co } \pi}$ is a semisimple braided Hopf algebra over $\mathbb{Z}_{p}$. The coalgebra $R$ is cocommutative, by Lemma 4.7, because $R \simeq H^{*} / H^{*} k G\left(H^{*}\right)^{+}$as coalgebras. Since $p \nmid 1+n p=\operatorname{dim} R$ then by [Sommerhäuser 2002, Proposition 7.2], $R$ is trivial. Therefore, by [Natale 2007b, Proposition 4.6.1], $H^{*}$ fits into an abelian central exact sequence

$$
k \rightarrow k \mathbb{Z}_{p} \rightarrow H^{*} \rightarrow R \rightarrow k
$$

Now, since the extension is abelian, there is a group $F$ such that $R \simeq k F$. It follows from Corollary 4.4 that $H^{*}$ is nilpotent.

Proposition 4.9. Suppose $H$ is quasitriangular. Then $G\left(H^{*}\right) \subseteq Z\left(H^{*}\right)$ and $H^{*}$ is nilpotent.

Proof. Consider the Drinfeld double $D(H)$. Since $H$ is quasitriangular, $G\left(H^{*}\right) \simeq \mathbb{Z}_{p}$ is isomorphic to a subgroup of $G\left(D(H)^{*}\right)$. Then $G\left(D(H)^{*}\right)$ has an element $g \# f$ of order $p$. We have

$$
G\left(D(H)^{*}\right) \simeq G(D(H)) \cap Z(D(H)) \subseteq G(D(H))=G\left(H^{*}\right) \times G(H) ;
$$


In particular, the element $f \# g \in G(D(H)) \cap Z(D(H))$ is of order $p$. If $g$ is of order $p$, then the proposition follows from Proposition 4.8. Thus we may assume that $g=1$. Then $f \in G\left(H^{*}\right) \cap Z\left(H^{*}\right)$ is of order $p$, implying that $G\left(H^{*}\right) \subseteq Z\left(H^{*}\right)$.

Therefore $H^{*}$ fits into an abelian central exact sequence

$$
k \rightarrow k^{\mathbb{Z}_{p}} \rightarrow H^{*} \rightarrow k F \rightarrow k,
$$

where $F$ is a finite group such that $k F \simeq H^{*} / H^{*}\left(k^{\mathbb{Z}_{p}}\right)^{+}$, by Lemma 4.7. In view of the assumption on the algebra structure of $H$, Corollary 4.4 implies that $H^{*}$ is nilpotent, as claimed.

4C. Results for the type $(1, p ; p, 1)$. We next discuss the case where $H$ is of type $(1, p ; p, 1)$ as an algebra (not necessarily quasitriangular). In particular, $\operatorname{dim} H=p(p+1)$ is even.

Notice that under this assumption, the category Rep $H$ is a near-group category with fusion rule given by the group $G=G\left(H^{*}\right) \simeq \mathbb{Z}_{p}$ and the integer $\kappa$ [Siehler 2003].

Let $\chi$ be the irreducible character of degree $p$. It follows that $\chi=\chi^{*}$ and $\chi g=\chi=g \chi$. Then

$$
\chi^{2}=\sum_{g \in G\left(H^{*}\right)} g+\kappa \chi .
$$

Taking degrees in the equation above we obtain $p^{2}=p+\kappa p$, which means that $\kappa=p-1$.

We shall use the following proposition. A more general statement will be proved in Theorem 6.2.

Proposition 4.10. Suppose $H$ is of type $(1, p ; p, 1)$ as an algebra. Then either

(i) $p=2$ and $H \simeq k \mathbb{S}_{3}$, or

(ii) $p=2^{\alpha}-1^{1}$ and $\operatorname{dim} H=2^{\alpha} p$.

In particular, $H$ is solvable.

Proof. By [Siehler 2003, Theorem 1.2], it follows that $G\left(H^{*}\right) \simeq \mathbb{Z}_{q^{\alpha}-1}$, for some prime $q$ and $\alpha \geq 1$. Therefore $p=q^{\alpha}-1$. If $q>2$, then $p=2$, which implies $H \simeq k \mathbb{S}_{3}$ is cocommutative. If $q=2$, then $p$ has the particular expression $p=2^{\alpha}-1$.

Hence $\operatorname{dim} H$ equals 6 or $p(p+1)=2^{\alpha} p$. By Burnside's theorem for fusion categories [Etingof et al. 2011, Theorem 1.6], $H$ is solvable.

Remark 4.11. Let $p$ be a prime number such that $p=2^{\alpha}-1$, as in Proposition 4.10. Consider the affine group $N$ of the field $\mathbb{F}_{2^{\alpha}}$, that is, $N$ is the semidirect product $\mathbb{F}_{2^{\alpha}} \rtimes \mathbb{F}_{2^{\alpha}}^{\times}$with respect to the natural action of $\mathbb{F}_{2^{\alpha}}^{\times}$on $\mathbb{F}_{2^{\alpha}}$. Then the group $N$ has the prescribed algebra type (see [Siehler 2003, §4.1]).

\footnotetext{
${ }^{1}$ Such a prime number is called a Mersenne prime; in particular $\alpha$ must be prime.
} 
Furthermore, suppose $p$ is (any) prime number, and $N$ is a group whose group algebra has algebra type $(1, p ; p, 1)$. Then $N$ has order $p(p+1)$ and it follows from the main result of [Seitz 1968] that either $p=2$ and $N \simeq \mathbb{S}_{3}$ or $p=2^{\alpha}-1$,

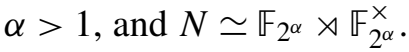

Proposition 4.12. Let $H$ be a semisimple Hopf algebra of type $(1, p ; p, 1)$ as an algebra. Then $G\left(H^{*}\right) \subseteq Z\left(H^{*}\right)$ and $H^{*}$ is nilpotent.

Proof. We have just proved in Proposition 4.10 that under this hypothesis $H$ is solvable. Since $\operatorname{Rep} D(H) \simeq Z(\operatorname{Rep} H)$, then $D(H)$ is also solvable [Etingof et al. 2011, Proposition 4.5(i)].

By [Etingof et al. 2011, Proposition 4.5(iv)], $D(H)$ has nontrivial representations of dimension 1, that is, $\left|G\left(D(H)^{*}\right)\right| \neq 1$. We have

$$
G\left(D(H)^{*}\right) \simeq G(D(H)) \cap Z(D(H)) \subseteq G(D(H))=G\left(H^{*}\right) \times G(H) ;
$$

see Section 2F.

We next argue as in the proof of Proposition 4.9. Consider an element $1 \neq$ $f \# g \in G(D(H)) \cap Z(D(H))$. If $f=1$, then $1 \neq g \in Z(H) \cap G(H)$. Therefore, $H^{*}$ fits into a cocentral extension $k \rightarrow K \rightarrow H^{*} \rightarrow k^{\langle g\rangle} \rightarrow k$, where $K$ is a proper normal Hopf subalgebra. The assumption on the algebra structure of $H$ implies that $K=k G\left(H^{*}\right)$. Thus $k G\left(H^{*}\right)$ is normal in $H^{*}$, and the extension is abelian, by Lemma 4.7. The proposition follows in this case from Proposition 4.3(i) and Corollary 4.4.

Thus we may assume that $f \neq 1$. In particular, $f$ has order $p$.

If $|f|=|g|=p=\left|G\left(H^{*}\right)\right|$, we have that $p|| G(H) \mid$. Then $G\left(H^{*}\right) \subseteq Z\left(H^{*}\right)$ and $H^{*}$ is nilpotent, by Proposition 4.8.

Otherwise, take $|g|=n$, with $p \neq n$. If $f^{n}=1$, then $p$ divides $n$ and thus $p$ divides $|G(H)|$. As before, we are done by Proposition 4.8.

If $f^{n} \neq 1$, then $f^{n} \# 1=\left(f^{n} \# g^{n}\right)=(f \# g)^{n} \in Z(D(H))$, which implies that $f^{n} \neq 1$ is central in $H^{*}$ and thus $G\left(H^{*}\right) \subseteq Z\left(H^{*}\right)$.

Therefore $H^{*}$ fits into an abelian central exact sequence

$$
k \rightarrow k^{\mathbb{Z}_{p}} \rightarrow H^{*} \rightarrow k F \rightarrow k,
$$

where $F$ is a finite group such that $k F \simeq H^{*} / H^{*}\left(k^{\mathbb{Z}_{p}}\right)^{+}$, by Lemma 4.7. In view of the assumption on the algebra structure of $H$, Corollary 4.4 implies that $H^{*}$ is nilpotent, as claimed.

Theorem 4.13. Let $H$ be a semisimple Hopf algebra of type $(1, p, p, 1)$ as an algebra. Then either $p=2$ and $H \simeq k \mathbb{S}_{3}$, or $H$ is isomorphic to a twisting of the group algebra $k N$, where $p=2^{\alpha}-1, \alpha>1$, and $N$ is the affine group of the field $\mathbb{F}_{2^{\alpha}}$. 
Proof. If $p=2$, then $\operatorname{dim} H=6$ and the result follows from [Masuoka 1995]. So suppose that $p$ is odd. By Propositions 4.12 and 4.10, $H^{*}$ fits into an abelian central exact sequence $k \rightarrow k^{\mathbb{Z}_{p}} \rightarrow H^{*} \rightarrow k F \rightarrow k$, where $F$ is a finite group of order $p+1=2^{\alpha}$. Then the action $\triangleleft: \mathbb{Z}_{p} \times F \rightarrow \mathbb{Z}_{p}$ is trivial, while the action $\triangleright: \mathbb{Z}_{p} \times F \rightarrow F$ is determined by an automorphism $\varphi \in$ Aut $F$ of order $p=2^{\alpha}-1$.

We first claim that the group $F$ must be abelian. By a result of P. Hall [Robinson 1982, (5.3.3)], since $F$ is a 2-group, the order of Aut $F$ divides the number $n 2^{(\alpha-r) r}$, where $n=|\mathrm{GL}(r, 2)|$ and $2^{r}$ equals the index in $F$ of the Frattini subgroup $\operatorname{Frat}(F)$ (which is defined as the intersection of all the maximal subgroups of $F$ [Robinson 1982, p. 135]). In particular, we have $r \leq \alpha$.

Since the order of $\varphi$ divides the order of Aut $F$ and $|\mathrm{GL}(r, 2)|=\left(2^{r}-1\right)\left(2^{r}-2\right)$ $\ldots\left(2^{r}-2^{r-1}\right)$, it follows that the prime $p=2^{\alpha}-1$ divides $2^{r}-1$, which means that $r=\alpha$ and, therefore, $\operatorname{Frat}(F)=1$.

Since $F$ is nilpotent (because it is a 2-group), a result of Wielandt [Robinson 1982, (5.2.16)] implies that $[F, F]$, the commutator subgroup of $F$, is a subgroup of the Frattini subgroup $\operatorname{Frat}(F)$. As we have just shown, we have $\operatorname{Frat}(F)=1$ in this case. Thus $[F, F]=1$ and therefore $F$ is abelian, as claimed.

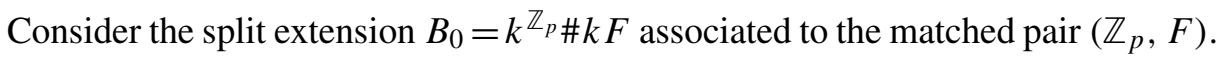
Since $F$ is abelian, $B_{0}$ (being a central extension) is commutative. This means that $B_{0}$ is isomorphic to $k^{N}$, where $N=F \rtimes \mathbb{Z}_{p}$.

Notice that $|F|=2^{\alpha}$ is relatively prime to $p$. It follows from [Natale 2007a, Proposition 5.22] and [Masuoka 2002, Proposition 3.1] that $H^{*}$ is obtained from

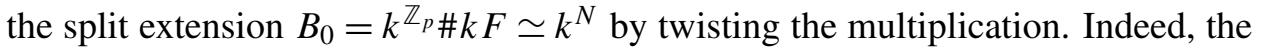
element representing the class of $H^{*}$ in the group $\operatorname{Opext}\left(k F, k^{\mathbb{Z}}\right)$ is the image of an element of $H^{2}\left(F, k^{\times}\right)$under the map $H^{2}\left(F, k^{\times}\right) \oplus H^{2}\left(\mathbb{Z}_{p}, k^{\times}\right) \simeq H^{2}\left(F, k^{\times}\right) \rightarrow$ $\operatorname{Opext}\left(k F, k^{\mathbb{Z}_{p}}\right)$ in the Kac exact sequence [Masuoka 2002, Theorem 1.10]. Then the claim follows from [Masuoka 2002, Proposition 3.1]. Dualizing, we get that $H$ is a twisting of the group algebra of the group $N$.

Finally, the assumption on the algebra structure of $H$ implies that $N$ is one of the claimed groups. See Remark 4.11.

Corollary 4.14. Let $H$ be a semisimple Hopf algebra of type $(1, p, p, 1)$ as an algebra. Then $\operatorname{Rep} H \simeq \operatorname{Rep} N$, where $N=\mathbb{S}_{3}$ or $N$ is the affine group of the field $\mathbb{F}_{2^{\alpha}}$, for some $\alpha>1$.

\section{Solvability}

Recall from [Etingof et al. 2011] that a fusion category $\mathscr{C}$ is called weakly grouptheoretical if it is Morita equivalent to a nilpotent fusion category. If, furthermore, $\mathscr{C}$ is Morita equivalent to a cyclically nilpotent fusion category, then $\mathscr{b}$ is called solvable. 
In other words, $\mathscr{C}$ is weakly group-theoretical (solvable) if there exists an indecomposable algebra $A$ in $\mathscr{C}$ such that the category ${ }_{A} \mathscr{C}_{A}$ of $A$-bimodules in $\mathscr{C}$ is a (cyclically) nilpotent fusion category.

Note that a group-theoretical fusion category is weakly group-theoretical.

On the other hand, the condition on $\mathscr{b}$ being solvable is equivalent to the existence of a sequence of fusion categories

$$
\mathscr{C}_{0}=\operatorname{Vec}_{k}, \mathscr{C}_{1}, \ldots, \mathscr{C}_{n}=\mathscr{C},
$$

such that $\mathscr{C}_{i}$ is obtained from $\mathscr{C}_{i-1}$ either by a $G_{i}$-equivariantization or as a $G_{i}$-extension, where $G_{1}, \ldots, G_{n}$ are cyclic groups of prime order. See [Etingof et al. 2011, Proposition 4.4].

If $G$ is a finite group and $\omega \in H^{3}\left(G, k^{\times}\right)$, we have that the categories $\mathscr{C}(G, \omega)$ and $\operatorname{Rep} G$ are solvable if and only if $G$ is solvable.

Let us call a semisimple Hopf algebra $H$ weakly group-theoretical or solvable if the category Rep $H$ is weakly group-theoretical or solvable, respectively.

5A. Solvability of an abelian extension. By [Etingof et al. 2011, Proposition 4.5(i)], solvability of a fusion category is preserved under Morita equivalence. Therefore, a group-theoretical fusion category $\mathscr{C}(G, \omega, F, \alpha)$ is solvable if and only if the group $G$ is solvable.

Remark 5.1. As a consequence of the Feit-Thompson theorem [1963], we get that if the order of $G$ is odd, then $\mathscr{b}(G, \omega, F, \alpha)$ is solvable. This fact generalizes to weakly group-theoretical fusion categories; see Proposition 7.1 below.

This implies the following characterization of the solvability of an abelian extension:

Corollary 5.2. Let $H$ be a semisimple Hopf algebra fitting into an abelian exact sequence (2-5); then $H$ is solvable if and only if $G=F \bowtie \Gamma$ is solvable.

In particular, if $H$ is solvable, then $F$ and $\Gamma$ are solvable.

A result of Wielandt [1958] implies that if the groups $\Gamma$ and $F$ are nilpotent, then $G$ is solvable. As a consequence, we get the following:

Corollary 5.3. Suppose $\Gamma$ and $F$ are nilpotent. Then $H$ is solvable.

Then, for instance, the abelian extensions in Proposition 4.3 are solvable.

Combining Corollary 5.3 with Lemma 4.1 and Remark 4.2, we get:

Corollary 5.4. Let

$$
\mathscr{C}=\mathscr{C}\left(G, \omega, \mathbb{Z}_{p}, \alpha\right) .
$$

Assume that $|G(\mathscr{C})|=p$. Then $\mathscr{C}$ is solvable. 


\section{Solvability from character degrees}

Let $p$ be a prime number. We study in this section fusion categories $\mathscr{b}$ such that c.d. $(\mathscr{C})=\{1, p\}$.

It is known that if $G$ is a finite group, then this assumption implies that the group $G$, and thus the category $\operatorname{Rep} G$, are solvable [Isaacs 1976].

Remark 6.1. If $H$ is any semisimple Hopf algebra such that c.d. $(H)=\{1, p\}$ and $G$ is any finite group, then the tensor product Hopf algebra $A=H \otimes k^{G}$ also satisfies that c.d. $(A)=\{1, p\}$ (since the irreducible modules of $A$ are tensor products of irreducible modules of $H$ and $k^{G}$ ).

But $A$ is not solvable unless $G$ is solvable; indeed, $k^{G}$ is a Hopf subalgebra as well as a quotient Hopf algebra of $A$.

Our aim in this section is to prove some structural results on $\mathscr{C}$, regarding solvability, under additional restrictions.

The following theorem generalizes Proposition 4.10.

Theorem 6.2. Let $\mathscr{C}$ be a near-group fusion category such that c.d. $(\mathscr{C})=\{1, p\}$. Then $\mathscr{C}$ is solvable.

Proof. In the notation of [Siehler 2003], let the fusion rules of $\mathscr{C}$ be given by the pair $(G, \kappa)$, where $G$ is the group of invertible objects of $\mathscr{C}$ and $\kappa$ is a nonnegative integer. Then $\operatorname{Irr}(\mathscr{C})=G \cup\{m\}$, with the relation

$$
m^{2}=\sum_{g \in G} g+\kappa m
$$

The assumption on c.d.( $\mathscr{C})$ implies that FPdim $m=p$. Hence FPdim $\mathscr{C}=$ $|G|+p^{2}$, and since $|G|=|G(\mathscr{C})|$ divides FPdim $\mathscr{C}$, we get that $|G|=p$ or $p^{2}$. (Note that, taking Frobenius-Perron dimensions in (6-1), we get that $G \neq 1$.)

If $|G|=p^{2}$, then $\kappa=0$ and $\mathscr{C}$ is a Tambara-Yamagami category [Tambara and Yamagami 1998]. Furthermore, $\mathscr{C}$ is a $\mathbb{Z}_{2}$-extension of a pointed category $\mathscr{C}(G, \omega)$. Then $\mathscr{C}$ is solvable in this case, by [Etingof et al. 2011, Proposition 4.5(i)].

Suppose that $|G|=p$. Then $\kappa=p-1$. As in the proof of Proposition 4.10, using [Siehler 2003, Theorem 1.2], we get that FPdim $\mathscr{C}=p(p+1)$ equals 6 or $p 2^{\alpha}$. Then $\mathscr{C}$ is solvable, by [Etingof et al. 2011, Theorem 1.6].

Our next result is the following theorem, for $\mathscr{C}=\operatorname{Rep} H$, which is a consequence of Proposition 4.9. A stronger version of this result will be given in Section 7B, under additional dimension restrictions.

Theorem 6.3. Suppose $H$ is of type $(1, p ; p, n)$ as an algebra. Assume in addition that $H$ is quasitriangular. Then $H$ is solvable. 
Proof. We have shown in Proposition 4.9 that $H^{*}$ is nilpotent. Moreover, by Lemma 4.7, $H$ fits into an abelian cocentral exact sequence

$$
k \rightarrow k^{F} \rightarrow H \rightarrow k \mathbb{Z}_{p} \rightarrow k,
$$

where $F$ is a nilpotent group. Therefore, $H$ is solvable, by Corollary 5.3.

In the remainder of this section, we restrict ourselves to the case where $\mathscr{C}=\operatorname{Rep} H$ for a semisimple Hopf algebra $H$.

6A. The case $\boldsymbol{p}=2$. Let $H$ be a semisimple Hopf algebra such that c.d. $(H) \subseteq$ $\{1,2\}$. By [Bichon and Natale 2011, Theorem 6.4], one of the following possibilities holds:

(i) there is a cocentral abelian exact sequence $k \rightarrow k^{F} \rightarrow H \rightarrow k \Gamma \rightarrow k$, where $F$ is a finite group and $\Gamma \simeq \mathbb{Z}_{2}{ }^{n}, n \geq 1$, or

(ii) there is a central exact sequence $k \rightarrow k^{U} \rightarrow H \rightarrow B \rightarrow k$, where $B=H_{\text {ad }}$ is a proper Hopf algebra quotient, and $U=U(\operatorname{Rep} H)$ is the universal grading group of the category of finite-dimensional $H$-modules.

In particular, if $H=H_{\text {ad }}$, then $H$ satisfies (i).

As a consequence of this result we have:

Theorem 6.4. Let $H$ be a semisimple Hopf algebra such that c.d. $(H) \subseteq\{1,2\}$. Then $H$ is weakly group-theoretical.

Moreover, if $H=H_{\mathrm{ad}}$, then $H$ is group-theoretical.

Proof. The assumption implies that $H$ satisfies (i) or (ii) above. If $H$ satisfies (i), then $H$ is group-theoretical, by Remark 2.4.

Otherwise, $H$ satisfies (ii), and then the category Rep $H$ is a $U$-extension of $\operatorname{Rep} B$, in view of Proposition 3.1. By an inductive argument, we may assume that $B$ is weakly group-theoretical (note that c.d. $(B) \subseteq\{1,2\}$ ). Therefore so is $H$, by [Etingof et al. 2011, Proposition 4.1].

We next discuss conditions that guarantee the solvability of $H$. The following result is proved in [Bichon and Natale 2011].

Proposition 6.5 [Bichon and Natale 2011, Proposition 6.8]. Suppose H is of type $(1,2 ; 2, n)$ as an algebra. Then $H$ is cocommutative.

The proposition implies that such a Hopf algebra $H$ is isomorphic to a group algebra $k G$ for some finite group $G$. By the assumption on the algebra structure of $H$, the group $G$, and then also $H$, are solvable.

The next lemma gives a sufficient condition for $H$ to be solvable.

Lemma 6.6. Suppose c.d. $(H) \subseteq\{1,2\}$ and $H=H_{\mathrm{ad}}$. Then $H$ is solvable if and only if the group $F$ in $(i)$ is solvable. 
Proof. Since $H=H_{\mathrm{ad}}$, then $H$ satisfies (i). Therefore $H$ is solvable if and only if the relevant factorizable group $G=F \bowtie \Gamma$ is solvable, by Corollary 5.2. Also, since the sequence (i) is cocentral, then $G$ is a semidirect product: $G=F \rtimes \Gamma$. This proves the lemma.

Remark 6.7. Suppose that $H$ has a faithful irreducible character $\chi$ of degree 2, such that $\chi \chi^{*}=\chi^{*} \chi$. Then it follows from [Bichon and Natale 2011, Theorem 3.5] that $H$ fits into a central abelian exact sequence $k \rightarrow k^{\mathbb{Z}_{m}} \rightarrow H \rightarrow k T \rightarrow k$, for some polyhedral group $T$ of even order and some $m \geq 1$. In particular, since c.d. $(H)=\{1,2\}$, then $T$ is necessarily cyclic or dihedral (see, for instance, [Bichon and Natale 2011, p. 10] for a description of the polyhedral groups and their character degrees). Therefore $H$ is solvable in this case.

The assumption on $\chi$ is satisfied in the case where $H$ is quasitriangular; hence the conclusion holds in this case. We shall show in the next subsection that every quasitriangular semisimple Hopf algebra with c.d. $(H) \subseteq\{1,2\}$ is also solvable.

We next prove some lemmas that will be useful in the next subsection.

Lemma 6.8. Suppose c.d. $(H) \subseteq\{1,2\}$ and let $K$ be a Hopf subalgebra or quotient Hopf algebra of $H$. Then c.d. $(K) \subseteq\{1,2\}$.

Proof. We only need to show the claim when $K \subseteq H$ is a Hopf subalgebra. In this case, the statement follows from surjectivity of the restriction functor Rep $H \rightarrow$ $\operatorname{Rep} K$.

The lemma has the following immediate consequence:

Corollary 6.9. If c.d. $(H) \subseteq\{1,2\}$, then the group $G(H)$ is solvable.

Lemma 6.10. Suppose c.d. $(H)$, c.d. $\left(H^{*}\right) \subseteq\{1,2\}$. Then $H$ is solvable.

Proof. By induction on the dimension of $H$.

Consider the universal grading group $U$ of the category $\operatorname{Rep} H$. Then $H^{*} \rightarrow k U$ is a quotient Hopf algebra and therefore c.d. $(U) \subseteq\{1,2\}$, by Lemma 6.8. This implies that the group $U$ is solvable.

Suppose first $H_{\mathrm{ad}} \neq H$. In view of Lemma 6.8, we also have c.d. $\left(H_{\mathrm{ad}}\right)$, c.d. $\left(H_{\mathrm{ad}}^{*}\right)$ $\subseteq\{1,2\}$. By the inductive assumption $H_{\mathrm{ad}}$ is solvable. By [Etingof et al. 2011, Proposition 4.5(i)], $H$ is solvable, since Rep $H$ is a $U$-extension of $\operatorname{Rep} H_{\text {ad }}$.

It remains to consider the case where $H_{\mathrm{ad}}=H$. As pointed out at the beginning of this subsection, it follows from [Bichon and Natale 2011, Theorem 6.4] that in this case $H$ satisfies condition (i), that is, $H$ fits into a cocentral abelian exact sequence $k \rightarrow k^{F} \rightarrow H \rightarrow k \Gamma \rightarrow k$, with $|\Gamma|>1$ and $\Gamma$ abelian.

In particular, $k^{\Gamma} \subseteq H^{*}$ is a nontrivial central Hopf subalgebra, implying that $H^{*} \neq H_{\mathrm{ad}}^{*}$. The inductive assumption implies, as before, that $H_{\mathrm{ad}}^{*}$ and thus also $H^{*}$ is solvable. Then $H$ is too. 
6B. The quasitriangular case. We shall assume in this subsection that $H$ is quasitriangular. Let $R \in H \otimes H$ be an $R$-matrix. We keep the notation of Section $2 \mathrm{~F}$.

Remark 6.11. Since the category $\operatorname{Rep} H$ is braided, then the universal grading group $U=U(\operatorname{Rep} H)$ is abelian (and, in particular, solvable).

The following is the main result of this subsection.

Theorem 6.12. Let $H$ be a quasitriangular semisimple Hopf algebra such that c.d. $(H) \subseteq\{1,2\}$. Then $H$ is solvable.

Proof. If c.d. $(H)=\{1\}$, then $H$ is commutative and, because it is quasitriangular, isomorphic to the group algebra of an abelian group. Hence we may assume that c.d. $(H)=\{1,2\}$.

Consider the Hopf subalgebras $H_{+}, H_{-} \subseteq H$. By Lemma 6.8, we have c.d. $\left(H_{+}\right)$, c.d. $\left(H_{-}\right) \subseteq\{1,2\}$. Then c.d. $\left(H_{-}\right)$, c.d. $\left(H_{-}^{*}\right) \subseteq\{1,2\}$, since $\left(H_{-}^{*}\right)^{\text {cop }} \simeq H_{+}$.

By Lemma 6.10, $H_{-}$is solvable. Therefore the Drinfeld double $D\left(H_{-}\right)$and its homomorphic image $H_{R}$ are also solvable.

We may thus assume that $H_{R} \subsetneq H$.

Observe that, being a quotient of $H, H_{\mathrm{ad}}$ is also quasitriangular and satisfies c.d. $\left(H_{\mathrm{ad}}\right) \subseteq\{1,2\}$. Hence, by induction, we may also assume that $H=H_{\mathrm{ad}}$, and, in particular, $G(H) \cap Z(H)=1$. Indeed, $\operatorname{Rep} H$ is a $U$-extension of $\operatorname{Rep} H_{\mathrm{ad}}$ and the group $U$ is abelian, as pointed out before.

Therefore $H$ fits into a cocentral abelian exact sequence $k \rightarrow k^{F} \rightarrow H \rightarrow k \Gamma \rightarrow k$, where $1 \neq \Gamma$ is elementary abelian of exponent 2 .

In view of Lemma 6.6, it will be enough to show that the group $F$ is solvable.

We have $\widehat{\Gamma} \subseteq G\left(H^{*}\right) \cap Z\left(H^{*}\right)$. By [Radford 1992, Proposition 3],

$$
f_{R_{21}}\left(G\left(H^{*}\right) \cap Z\left(H^{*}\right)\right) \subseteq G(H) \cap Z(H) .
$$

Hence we may assume that $f_{R_{21}} \mid \widehat{\Gamma}=1$ and similarly $f_{R} \mid \widehat{\Gamma}=1$. Thus $f_{R}$ and $f_{R_{21}}$ factorize through the quotient $H^{*} / H^{*}(k \widehat{\Gamma})^{+} \simeq k F$.

Therefore $H_{+}=f_{R}\left(H^{*}\right)$ and $H_{-}=f_{R_{21}}\left(H^{*}\right)$ are cocommutative. (Then they are also commutative, since $H_{+} \simeq H_{-}^{* \text { cop }}$.) In particular, $H_{R}=H_{+} H_{-}$is cocommutative. Hence $\Phi_{R}\left(H^{*}\right) \subseteq H_{R} \subseteq k G(H)$.

By [Natale 2006, Theorem 4.11], $K=\Phi_{R}\left(H^{*}\right)$ is a commutative (and cocommutative) normal Hopf subalgebra, which is necessarily solvable, since $H_{R}$ is. In addition, $\Phi_{R}\left(H^{*}\right) \simeq k T$, where $T \subseteq G(H)$ is an abelian subgroup [Natale 2006, Example 2.1], and there is an exact sequence of Hopf algebras

$$
k \rightarrow k T \rightarrow H \stackrel{\pi}{\rightarrow} \bar{H} \rightarrow k,
$$

where $\bar{H}$ is a certain (canonical) triangular Hopf algebra.

Since $\bar{H}$ is triangular, $\bar{H} \simeq(k L)^{J}$ is a twisting of the group algebra of some 
finite group $L$. Because c.d. $(L)=$ c.d. $(\bar{H}) \subseteq\{1,2\}, L$ must be solvable. Hence $\bar{H}$ is solvable, since $\operatorname{Rep} \bar{H} \simeq \operatorname{Rep} L$.

The map $\pi: H \rightarrow \bar{H}$ induces, by restriction to the Hopf subalgebra $k^{F} \subseteq H$, an exact sequence

$$
k \rightarrow k T \cap k^{F} \rightarrow k \stackrel{\left.\pi\right|_{k} F}{\rightarrow} \pi\left(k^{F}\right) \rightarrow k
$$

We have $k T \cap k^{F}=k^{\bar{F}}$ and $\pi\left(k^{F}\right)=k^{S}$, where $\bar{F}$ and $S$ are a quotient and a subgroup of $F$, respectively, in such a way that the exact sequence above corresponds to an exact sequence of groups

$$
1 \rightarrow S \rightarrow F \rightarrow \bar{F} \rightarrow 1
$$

Now, $\bar{F}$ is abelian, because $k^{\bar{F}}=k T \cap k^{F}$ is cocommutative, and $S$ is solvable, because $k^{S}$ is a Hopf subalgebra of $\bar{H}$. Therefore $F$ is solvable. This implies that $H$ is solvable and finishes the proof of the theorem.

\section{Odd-dimensional fusion categories}

In this section, $p$ will be a prime number. Let $\mathscr{C}$ be a fusion category over $k$. Recall that the set of irreducible degrees of $\mathscr{C}$ was defined as

$$
\text { c.d. }(\mathscr{C})=\{\operatorname{FPdim} x \mid x \in \operatorname{Irr} \mathscr{C}\} .
$$

The fusion categories that we shall consider in this section are all integral, that is, the Frobenius-Perron dimensions of objects of $\mathscr{C}$ are (natural) integers. By [Etingof et al. 2005, Theorem 8.33], $\mathscr{C}$ is isomorphic to the category of representations of some finite-dimensional semisimple quasi-Hopf algebra.

7A. Odd-dimensional weakly group-theoretical fusion categories. The following result is a consequence of the Feit-Thompson theorem [1963].

Proposition 7.1. Let $\mathscr{C}$ be a weakly group-theoretical fusion category and assume that FPdim $\mathscr{C}$ is an odd integer. Then $\mathscr{C}$ is solvable.

Note that since FPdim $\mathscr{b}$ is an odd integer, the fusion category $\mathscr{b}$ is integral. See [Drinfeld et al. 2010, Corollary 2.22].

Proof. By definition, $\mathscr{C}$ is Morita equivalent to a nilpotent fusion category. Then, by [Etingof et al. 2011, Proposition 4.5(i)], it will be enough to show that a nilpotent fusion category of odd Frobenius-Perron dimension is solvable. So, assume that $\mathscr{C}$ is nilpotent, so that $\mathscr{C}$ is a $G$-extension of a fusion subcategory $\widetilde{C}$, with $|G|>1$. In particular, FPdim $\mathscr{C}=|G|$ FPdim $\widetilde{\mathscr{C}}$. Hence FPdim $\widetilde{\mathscr{C}}$ and the order of $G$ are both odd, and FPdim $\widetilde{\mathscr{C}}<$ FPdim $\mathscr{C}$. The proposition follows by induction, since $G$ is solvable by the Feit-Thompson theorem; see [Etingof et al. 2011, Proposition 4.5(i)]. 
7B. Braided fusion categories. We shall need the following lemma whose proof is contained in the proof of [Etingof et al. 2011, Proposition 6.2(i)]. We include a sketch of the argument for the sake of completeness.

Lemma 7.2. Let $\mathscr{b}$ be a fusion category and let $G$ be a finite group acting on $\mathscr{b}$ by tensor autoequivalences. Assume c.d. $\left(\mathscr{C}^{G}\right) \subseteq\left\{p^{m}: m \geq 0\right\}$, where $p$ is a prime number. Then c.d. $(\mathscr{C}) \subseteq\left\{p^{m}: m \geq 0\right\}$.

Proof. Regard $\mathscr{C}$ as an indecomposable module category over itself via tensor product, and similarly for $\mathscr{C}^{G}$. Let $Y$ be a simple object of $\mathscr{C}$. Since the forgetful functor $F: \mathscr{C}^{G} \rightarrow \mathscr{C}$ is surjective, $Y$ is a simple constituent of $F(X)$, for some simple object $X$ of $\mathscr{C}^{G}$.

Since $F$ is a tensor functor, we have FPdim $X=F P d i m ~ F(X)$. By formula (7) in [Etingof et al. 2011, Proof of Proposition 6.2],

$$
\operatorname{FPdim}(X)=\operatorname{deg}(\pi)\left[G: G_{Y}\right] \operatorname{FPdim} Y,
$$

where $G_{Y} \subseteq G$ is the stabilizer of $Y$ and $\pi$ is an irreducible representation of $G_{Y}$ associated to $X$. Therefore FPdim $Y$ divides FPdim $X$.

The assumption on $\mathscr{C}^{G}$ implies that FPdim $X$ is a power of $p$. Then so is FPdim $Y$. This proves the lemma.

Theorem 7.3. Let $\mathscr{C}$ be a braided fusion category such that $\mathrm{c}$.d.( $(\mathscr{C}) \subseteq\left\{p^{m}: m \geq 0\right\}$, where $p$ is a prime number. Assume that $\mathrm{FPdim} \mathscr{C}$ is odd. Then $\mathscr{C}$ is solvable.

Proof. By induction on FPdim $\mathscr{C}$. (The Frobenius-Perron dimension of a fusion subcategory of $\mathscr{C}$ divides the dimension of $\mathscr{C}$ [Etingof et al. 2005, Proposition 8.15], and the same is true for the Frobenius-Perron dimension of a fusion category $\mathscr{D}$ such that there exists a surjective tensor functor $\mathscr{C} \rightarrow \mathscr{D}$ [Etingof et al. 2005, Corollary 8.11]. Thus these fusion categories are odd-dimensional as well.) If c.d. $(\mathscr{C})=\{1\}$, then $\mathscr{C}$ is pointed. Then $\mathscr{C} \simeq \mathscr{C}(G, \omega)$ for some abelian group $G$ and some 3-cocycle $\omega$ on $G$. Then $\mathscr{C}$ is solvable, by [Etingof et al. 2011, Proposition 4.5(ii)].

Suppose next that $\mathscr{C}$ is not pointed. Then all noninvertible objects in $\mathscr{C}$ have Frobenius-Perron dimension $p^{m}$, for some $m \geq 1$. Consider the group $G(\mathscr{C})$ of invertible objects of $\mathscr{C}$. Then $G(\mathscr{C})$ is abelian and $G(\mathscr{C}) \neq 1$, as follows by taking Frobenius-Perron dimensions in a decomposition of the tensor product $X \otimes X^{*}$, for some simple noninvertible object $X$.

Let us regard $\mathscr{C}$ as a premodular fusion category with respect to its canonical spherical structure (as FPdim $\mathscr{C}$ is an integer). Then $\mathscr{C}$ is modularizable, in view of [Bruguières and Natale 2011, Lemma 7.2].

Let $\widetilde{\mathscr{C}}$ be its modularization, which is a modular category over $k$. Then $\mathscr{b}$ is an equivariantization $\mathscr{C} \simeq \widetilde{\mathscr{C}}^{G}$ with respect to the action of a certain group $G$ on $\widetilde{\mathscr{C}}$ [Bruguières 2000]. (Indeed, the modularization functor $\mathscr{C} \rightarrow \widetilde{\mathscr{C}}$ gives rise to 
an exact sequence of fusion categories $\operatorname{Rep} G \rightarrow \mathscr{C} \rightarrow \widetilde{\mathscr{C}}$, which comes from an equivariantization; see [Bruguières and Natale 2011, Example 5.33].)

By construction of $G$, the category $\operatorname{Rep} G$ is the (tannakian) fusion subcategory of transparent objects in $\mathscr{C}$. Therefore there is an embedding of braided fusion categories $\operatorname{Rep} G \subseteq \mathscr{C}$. In particular, the order of $G$ is odd, implying that $G$ is solvable.

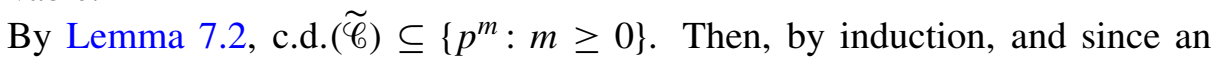
equivariantization of a solvable fusion category under the action of a solvable group is again solvable, we may and shall assume in what follows that $\mathscr{C}=\widetilde{\mathscr{C}}$ is modular.

It is shown in [Gelaki and Nikshych 2008, Theorem 6.2] that the universal grading group $U(\mathscr{b})$ is (abelian and) isomorphic to the group $\widehat{G(\mathscr{b})}$ of characters of $G(\mathscr{C})$. In particular, $U(\mathscr{C}) \neq 1$. On the other hand, $\mathscr{C}$ is a $U(\mathscr{b})$-extension of its fusion subcategory $\mathscr{C}_{\text {ad }}$. Since also c.d. $\left(\mathscr{C}_{\mathrm{ad}}\right) \subseteq\left\{p^{m}: m \geq 0\right\}$, then $\mathscr{C}_{\text {ad }}$ is solvable, by induction. Therefore $\mathscr{C}$ is solvable, as claimed.

\section{References}

[Bakalov and Kirillov 2001] B. Bakalov and A. Kirillov, Jr., Lectures on tensor categories and modular functors, University Lecture Series 21, American Mathematical Society, Providence, RI, 2001. MR 2002d:18003 Zbl 0965.18002

[Bichon and Natale 2011] J. Bichon and S. Natale, "Hopf algebra deformations of binary polyhedral groups”, Transform. Groups 16:2 (2011), 339-374. MR 2012g:16066 Zbl 1238.16024

[Bruguières 2000] A. Bruguières, "Catégories prémodulaires, modularisations et invariants des variétés de dimension 3”, Math. Ann. 316:2 (2000), 215-236. MR 2001d:18009 Zbl 0943.18004

[Bruguières and Natale 2011] A. Bruguières and S. Natale, "Exact sequences of tensor categories", Int. Math. Res. Not. 2011 (2011), 5644-5705. MR 2863377 Zbl 05994502

[Drinfeld et al. 2010] V. Drinfeld, S. Gelaki, D. Nikshych, and V. Ostrik, "On braided fusion categories, I”, Selecta Math. (N.S.) 16:1 (2010), 1-119. MR 2011e:18015 Zbl 1201.18005

[Etingof and Gelaki 2003] P. Etingof and S. Gelaki, "The classification of finite-dimensional triangular Hopf algebras over an algebraically closed field of characteristic 0", Mosc. Math. J. 3:1 (2003), 37-43, 258. MR 2004i:16052 Zbl 1062.16043

[Etingof et al. 2005] P. Etingof, D. Nikshych, and V. Ostrik, "On fusion categories", Ann. of Math.

(2) 162:2 (2005), 581-642. MR 2006m:16051 Zbl 1125.16025

[Etingof et al. 2011] P. Etingof, D. Nikshych, and V. Ostrik, "Weakly group-theoretical and solvable fusion categories", Adv. Math. 226:1 (2011), 176-205. MR 2012g:18010 Zbl 1210.18009

[Feit and Thompson 1963] W. Feit and J. G. Thompson, "Solvability of groups of odd order", Pacific J. Math. 13 (1963), 775-1029. MR 29 \#3538 Zbl 0124.26402

[Gelaki and Naidu 2009] S. Gelaki and D. Naidu, "Some properties of group-theoretical categories", J. Algebra 322:8 (2009), 2631-2641. MR 2011d:20099 Zbl 1209.18007

[Gelaki and Nikshych 2008] S. Gelaki and D. Nikshych, "Nilpotent fusion categories", Adv. Math. 217:3 (2008), 1053-1071. MR 2009b:18015 Zbl 1168.18004

[Isaacs 1976] I. M. Isaacs, Character theory of finite groups, Pure and Applied Mathematics 69, Academic, New York, 1976. MR 57 \#417 Zbl 0337.20005 
[Izumi and Kosaki 2002] M. Izumi and H. Kosaki, Kac algebras arising from composition of subfactors: general theory and classification, Mem. Amer. Math. Soc. 158, 2002. MR 2004b:46090 Zbl 1001.46040

[Kashina et al. 2002] Y. Kashina, G. Mason, and S. Montgomery, "Computing the Frobenius-Schur indicator for abelian extensions of Hopf algebras", J. Algebra 251:2 (2002), 888-913. MR 2003f: 16061 Zbl 1012.16040

[Masuoka 1995] A. Masuoka, "Semisimple Hopf algebras of dimension 6, 8", Israel J. Math. 92:1-3 (1995), 361-373. MR 96j:16045 Zbl 0839.16036

[Masuoka 1999] A. Masuoka, "Extensions and cohomology of Hopf algebras, Lie bialgebras", pp. 131-149 in Proceedings of the 31st symposium on ring theory and representation theory and JapanKorea ring theory and representation theory seminar (Osaka, 1998), edited by K. Nishida and M. Sato, Shinshu University, Matsumoto, 1999. MR 1812913 Zbl 1222.16022

[Masuoka 2002] A. Masuoka, "Hopf algebra extensions and cohomology", pp. 167-209 in New directions in Hopf algebras, edited by S. Montgomery and H.-J. Schneider, Math. Sci. Res. Inst. Publ. 43, Cambridge University Press, 2002. MR 2003d:16050 Zbl 1011.16024

[Montgomery and Witherspoon 1998] S. Montgomery and S. J. Witherspoon, "Irreducible representations of crossed products", J. Pure Appl. Algebra 129:3 (1998), 315-326. MR 99d:16030 Zbl 0932.16039

[Natale 1999] S. Natale, "On semisimple Hopf algebras of dimension $p q^{2}$ ", J. Algebra 221:1 (1999), 242-278. MR 2000k:16050 Zbl 0942.16045

[Natale 2003] S. Natale, "On group theoretical Hopf algebras and exact factorizations of finite groups”, J. Algebra 270:1 (2003), 199-211. MR 2004k:16102 Zbl 1040.16027

[Natale 2005] S. Natale, "Frobenius-Schur indicators for a class of fusion categories", Pacific J. Math. 221:2 (2005), 353-377. MR 2007j:16070 Zbl 1108.16035

[Natale 2006] S. Natale, “ $R$-matrices and Hopf algebra quotients", Int. Math. Res. Not. 2006:18 (2006), Art. ID 47182. MR 2007g:16056 Zbl 1113.16043

[Natale 2007a] S. Natale, "On the exponent of tensor categories coming from finite groups", Israel J. Math. 162 (2007), 253-273. MR 2008k:16003 Zbl 1152.16029

[Natale 2007b] S. Natale, Semisolvability of semisimple Hopf algebras of low dimension, Mem. Amer. Math. Soc. 186, 2007. MR 2008b:16066 Zbl 1185.16033

[Natale 2011] S. Natale, "Semisimple Hopf algebras and their representations", Publ. Mat. Uruguay 12 (2011), 123-167.

[Nichols and Richmond 1996] W. D. Nichols and M. B. Richmond, "The Grothendieck group of a Hopf algebra”, J. Pure Appl. Algebra 106:3 (1996), 297-306. MR 97a:16075 Zbl 0848.16034

[Nichols and Zoeller 1989] W. D. Nichols and M. B. Zoeller, "A Hopf algebra freeness theorem”, Amer. J. Math. 111:2 (1989), 381-385. MR 90c:16008 Zbl 0672.16006

[Ostrik 2003] V. Ostrik, "Module categories over the Drinfeld double of a finite group", Int. Math. Res. Not. 2003:27 (2003), 1507-1520. MR 2004h:18005 Zbl 1044.18005

[Radford 1992] D. E. Radford, "On the antipode of a quasitriangular Hopf algebra”, J. Algebra 151:1 (1992), 1-11. MR 93i:16053 Zbl 0767.16016

[Radford 1993] D. E. Radford, "Minimal quasitriangular Hopf algebras", J. Algebra 157:2 (1993), 285-315. MR 94c:16052 Zbl 0787.16028

[Reshetikhin and Semenov-Tian-Shansky 1988] N. Reshetikhin and M. Semenov-Tian-Shansky, "Quantum $R$-matrices and factorization problems", J. Geom. Phys. 5:4 (1988), 533-550. MR 92g: 17019 Zbl 0711.17008 
[Robinson 1982] D. J. S. Robinson, A course in the theory of groups, Graduate Texts in Mathematics 80, Springer, New York, 1982. MR 84k:20001 Zbl 0483.20001

[Schneider 2001] H.-J. Schneider, "Some properties of factorizable Hopf algebras", Proc. Amer. Math. Soc. 129:7 (2001), 1891-1898. MR 2002a:16047 Zbl 0982.16031

[Seitz 1968] G. Seitz, "Finite groups having only one irreducible representation of degree greater than one”, Proc. Amer. Math. Soc. 19 (1968), 459-461. MR 36 \#5212 Zbl 0244.20010

[Siehler 2003] J. Siehler, "Near-group categories", Algebr. Geom. Topol. 3 (2003), 719-775. MR 2005a:18013 Zbl 1033.18004

[Sommerhäuser 2002] Y. Sommerhäuser, Yetter-Drinfel'd Hopf algebras over groups of prime order, Lecture Notes in Mathematics 1789, Springer, Berlin, 2002. MR 2003m:16054 Zbl 1006.16055

[Tambara and Yamagami 1998] D. Tambara and S. Yamagami, "Tensor categories with fusion rules of self-duality for finite abelian groups”, J. Algebra 209:2 (1998), 692-707. MR 2000b:18013 Zbl 0923.46052

[Wielandt 1958] H. Wielandt, "Über Produkte von nilpotenten Gruppen”, Illinois J. Math. 2 (1958), 611-618. MR 25 \#121 Zbl 0084.02904

[Zhu 1993] S. L. Zhu, “On finite-dimensional semisimple Hopf algebras", Comm. Algebra 21:11 (1993), 3871-3885. MR 95d:16057 Zbl 0802.16037

Communicated by Susan Montgomery

Received 2011-03-11 Revised 2011-04-01 Accepted 2011-10-28

natale@famaf.unc.edu.ar

plavnik@famaf.unc.edu.ar
Facultad de Matemática, Astronomía y Física, Universidad Nacional de Córdoba, Medina Allende $s / n$, Ciudad Universitaria, 5000 Córdoba, Argentina http://www.famaf.unc.edu.ar/ natale

Facultad de Matemática, Astronomía y Física, Universidad Nacional de Córdoba, Medina Allende s/n, Ciudad Universitaria, 5000 Córdoba, Argentina 


\section{Algebra \& Number Theory}

msp.berkeley.edu/ant

\section{EDITORS}

MANAGING EDITOR

Bjorn Poonen

Massachusetts Institute of Technology

Cambridge, USA

\author{
EDITORIAL BOARD CHAIR \\ David Eisenbud \\ University of California \\ Berkeley, USA
}

\section{BOARD OF EDITORS}

Georgia Benkart

Dave Benson

Richard E. Borcherds

John H. Coates

J-L. Colliot-Thélène

Brian D. Conrad

Hélène Esnault

Hubert Flenner

Edward Frenkel

Andrew Granville

Joseph Gubeladze

Ehud Hrushovski

Craig Huneke

Mikhail Kapranov

Yujiro Kawamata

János Kollár

Yuri Manin

Barry Mazur

Philippe Michel
University of Wisconsin, Madison, USA

University of Aberdeen, Scotland

University of California, Berkeley, USA

University of Cambridge, UK

CNRS, Université Paris-Sud, France

University of Michigan, USA

Universität Duisburg-Essen, Germany

Ruhr-Universität, Germany

University of California, Berkeley, USA

Université de Montréal, Canada

San Francisco State University, USA

Hebrew University, Israel

University of Kansas, USA

Yale University, USA

University of Tokyo, Japan

Princeton University, USA

Northwestern University, USA

Harvard University, USA

École Polytechnique Fédérale de Lausanne
Susan Montgomery

Shigefumi Mori

Raman Parimala

Jonathan Pila

Victor Reiner

Karl Rubin

Peter Sarnak

Joseph H. Silverman

Michael Singer

Vasudevan Srinivas

J. Toby Stafford

Bernd Sturmfels

Richard Taylor

Ravi Vakil

Michel van den Bergh

Marie-France Vignéras

Kei-Ichi Watanabe

Andrei Zelevinsky

Efim Zelmanov
University of Southern California, USA

RIMS, Kyoto University, Japan

Emory University, USA

University of Oxford, UK

University of Minnesota, USA

University of California, Irvine, USA

Princeton University, USA

Brown University, USA

North Carolina State University, USA

Tata Inst. of Fund. Research, India

University of Michigan, USA

University of California, Berkeley, USA

Harvard University, USA

Stanford University, USA

Hasselt University, Belgium

Université Paris VII, France

Nihon University, Japan

Northeastern University, USA

University of California, San Diego, USA

\section{PRODUCTION}

contact@msp.org

Silvio Levy, Scientific Editor

See inside back cover or www.jant.org for submission instructions.

The subscription price for 2012 is US \$175/year for the electronic version, and \$275/year (+ \$40 shipping outside the US) for print and electronic. Subscriptions, requests for back issues from the last three years and changes of subscribers address should be sent to Mathematical Sciences Publishers, Department of Mathematics, University of California, Berkeley, CA 94720-3840, USA.

Algebra \& Number Theory (ISSN 1937-0652) at Mathematical Sciences Publishers, Department of Mathematics, University of California, Berkeley, CA 94720-3840 is published continuously online. Periodical rate postage paid at Berkeley, CA 94704, and additional mailing offices.

ANT peer review and production are managed by EditFLOW ${ }^{\circledR}$ from Mathematical Sciences Publishers.

PUBLISHED BY

mathematical sciences publishers

http://msp.org/

A NON-PROFIT CORPORATION

Typeset in IATEX

Copyright $@ 2012$ by Mathematical Sciences Publishers 


\section{Algebra \& Number Theory}

Volume $6 \quad$ No. $6 \quad 2012$

The smallest prime that does not split completely in a number field

XIANNAN LI

On the geometric realization of the inner product and canonical basis for quantum affine

1097 $\mathfrak{s l}_{n}$

\section{KEVIN MCGERTY}

Combinatorics of the tropical Torelli map

MELODY CHAN

Sonia Natale and Julia Yael PlavniK

Cusp form motives and admissible $G$-covers

DAN PETERSEN

Ideals of degree one contribute most of the height

AARON LEVIN and DAVID MCKINNON

Torsion des modules de Drinfeld de rang 2 et formes modulaires de Drinfeld 